\title{
Contact Problems of Functionally Graded Materials
}

Modern technological developments require innovative materials to meet ever increasing performance demands. An example of this is functionally graded materials (FGMs) whose material composition or micro-structure varies continually within the volume in a pre-defined way. In this manner, the material properties can be set (possibly independently of each other) to the optimal values. A controlled gradient of the elasticity modulus has been proven to lead to a greater resistance to contact and friction damage (Suresh 2001). For instance, Hertzian cone cracks are suppressed due to the reduction of the maximum tensile stresses in the surface (Jitcharoen et al. 1998) and the wear resistance is increased (Suresh et al. 1999). In mechanical engineering, components such as cutting tools, gears, and parts of roller bearings or turbine blades are made from FGMs. This is just a small sample of the constantly expanding range of applications of FGMs (Miyamoto et al. 1999). In the biomedical arena, for endoprosthetics in particular, the use of FGMs in artificial knee and hip joints is intended to improve biocompatibility and reduce wear, extending the service life of the endoprosthesis and, thereby, increasing quality of life (Sola et al. 2016). A high degree of biocompatibility is also essential for dental implants (Mehrali et al. 2013).

Many insects and animals such as geckos possess highly effective attachment devices, allowing them to stick to and move on surfaces of widely varying ranges of roughness and topographies. This has inspired a great amount of research into the adhesion of biological structures with the aim of manufacturing artificial surfaces with similar adhesive properties (Boesel et al. 2010; Gorb et al. 2007). In fact, many biological structures exhibit functional material gradients which serve to optimize the adhesive properties (Peisker et al. 2013; Liu et al. 2017). Furthermore, the adhesive properties of FGMs are of importance to the fields of innovative nanoelectromechanical and micro-electromechanical systems (NEMS, MEMS).

Although the term "functionally graded material" was only coined in 1986 (Miyamoto et al. 1999), analytical and experimental research of their contact mechanical behavior had already been conducted much earlier. The origin lies in the field of geomechanics where the influence of the elasticity modulus, which increases with the depth of the soil foundation, on the stresses and displacements was 
of particular interest (Fröhlich 1934; Holl 1940). Over the years, different functions for the varying elasticity modulus were in use. On this issue, the works of Selvadurai (2007) and Aleynikov (2011) offer a good overview and we can safely skip over providing a complete list of references. In nearly all cases, the calculations are exceedingly complex and only allow numerical solutions. The majority of the publications deal with an exponential increase or a power-law dependent increase of the elasticity modulus. For an exponential change of the elasticity modulus, only approximate solutions exist (so far) (Giannakopoulos and Suresh 1997). However, contact problems of elastically inhomogeneous materials that obey the law

$$
E(z)=E_{0}\left(\frac{z}{c_{0}}\right)^{k} \quad \text { with }-1<k<1
$$

can be solved exactly in an analytical manner. The Poisson's ratio is assumed to be constant. Due to the fact that the elasticity modulus of the foundation always increases with the depth, law (9.1) was initially restricted to positive exponents. The first solutions go back to Holl (1940) and Rostovtsev (1961), who imposed the additional restriction $v=1 /(2+k)$. Complete solutions of frictionless normal contacts were provided by Booker et al. (1985) and Giannakopoulos and Suresh (1997). A special case of (9.1) is the famous Gibson medium: the linear-inhomogeneous, incompressible half-space $(k \rightarrow 1, v \rightarrow 1 / 2)$. Gibson (1967) managed to prove that such a medium behaves like a Winkler foundation (see also the work of Awojobi and Gibson 1973). If the solutions of the contact without adhesion are known, the work required to transfer that understanding to the adhesive normal contact between graded materials is not too great. Still, for an elastic inhomogeneity according to (9.1), the problem was only solved in the previous decade and remains the subject of current research (Chen et al. 2009; Jin et al. 2013, 2016; Willert 2018). However, all of these works assume a positive value of the exponent, which restricts their theoretical application to the class of graded materials where the elasticity modulus grows with increasing depth. Yet, as already mentioned in this chapter, plenty of practical applications exist (e.g., cutting tools, dental implants) which require a hard surface along with a softer core. A careful examination of the literature revealed that the theory is equally valid for negative exponents $-1<k<0$ (Rostovtsev 1964; Fabrikant and Sankar 1984). Although law (9.1) now permits the representation of positive and negative material gradients, and barring the special case of $k=0$, the law remains physically unrealistic for homogeneous materials due to the vanishing or infinitely large elasticity modulus at the surface and at infinite depth, respectively. Nevertheless, FEM calculations by Lee et al. (2009) confirmed that it yields qualitatively correct results for functional gradients, if the modulus is described piecewise by a power-law.

Analytical solutions of tangential contacts between materials exhibiting an elastic inhomogeneity according to (9.1) were only recently developed by Heß (2016b) and Heß and Popov (2016). Up until then, merely the plane tangential contact problem between a rigid, infinitely long cylinder and the elastically inhomogeneous half-space was considered completely solved (Giannakopoulos and Pallot 2000). In 
very recent times, the MDR-based theory of $\mathrm{He} ß$ and Popov enabled the fast calculation of even complicated impact problems of FGMs (Willert and Popov 2017a,b).

Following the order in which the applications of FGMs are presented in this chapter, we will now discuss frictionless normal contacts (Sect. 9.1), adhesive normal contacts (Sect. 9.2), and tangential contacts with partial slip (Sect. 9.3). We restrict the consideration to the elastic inhomogeneity given by (9.1). Furthermore, the parameter studies of the majority of the graphical solutions were conducted for positive exponents $0 \leq k<1$ only. Yet it must be expressly noted that all provided solutions are also valid for negative exponents $-1<k<0$. Additionally, our examination is usually limited to the contact of a rigid indenter and a functionally graded material. However, the theory is equally applicable to the contact between two elastically inhomogeneous bodies of the same exponent $k$ and of the same characteristic depths $c_{0}$. In this case, the Poisson's ratios $v_{i}$ and the elastic parameters $E_{0 i}$ are allowed to differ. It should also be noted that the special case $k=0$ yields many of the solutions for the contact problems of elastically homogeneous materials previously examined in Chaps. 2, 3, and 4.

\subsection{Frictionless Normal Contact Without Adhesion}

The frictionless normal contact between a rigid indenter of the shape $f(r)$ and a functionally graded material is shown in Fig. 9.1. Here, we differentiated between an elasticity modulus which drops with increasing depth (left) and one which rises with increasing depth (right). Law (9.1) has been demonstrated using a graphical representation.

\subsubsection{Basis for Calculation of the MDR}

For the solution of frictionless normal contacts without adhesion under consideration of the elastic inhomogeneity given by (9.1), we make use of the mapping rules and calculation formulas of the MDR developed by Heß (2016a). Accordingly, the
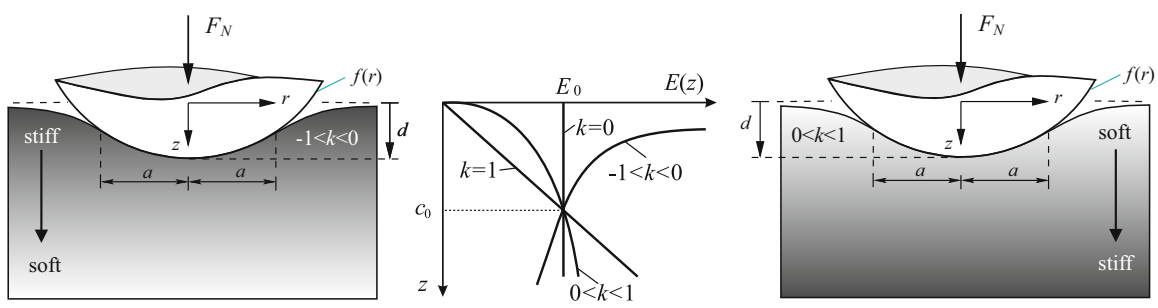

Fig. 9.1 Indentation of a rigid indenter with the profile $f(r)$ in an elastically inhomogeneous halfspace, whose elasticity modulus drops (left) or rises (right) with increasing depth according to the power-law (9.1), depending on whether the exponent $k$ is negative or positive 
Fig. 9.2 The equivalent substitute model for the normal contact between two elastically inhomogeneous half-spaces whose elasticity moduli satisfy condition $(9.1)$ while assuming that both media have equal exponents $k$

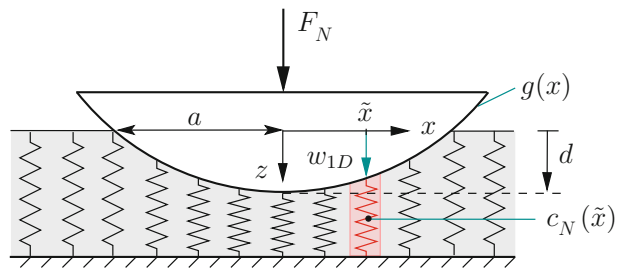

contact problem shown in Fig. 9.1 is equivalent to the indentation of a rigid, planar profile of the shape $g(x)$ in a one-dimensional Winkler foundation with respect to the relationships between normal force, contact radius, and indentation depth. This is valid for Winkler foundations whose stiffness depends on the coordinate $x$, which is expressed by a laterally varying foundation modulus $c_{N}(x)$ (spring stiffness divided by spring distance $\Delta x$ ). The equivalent substitute model is demonstrated in Fig. 9.2.

The planar profile $g(x)$, which is sometimes called the equivalent, one-dimensional substitute profile, is calculated according to:

$$
g(x)=|x|^{1-k} \int_{0}^{|x|} \frac{f^{\prime}(r)}{\left(x^{2}-r^{2}\right)^{\frac{1-k}{2}}} \mathrm{~d} r .
$$

The spring stiffness is given by equation:

$$
\Delta k_{z}(x)=c_{N}(x) \cdot \Delta x .
$$

Here, $c_{N}(x)$ refers to the foundation modulus (stiffness per unit of distance) which, in this case, depends on the distance to the contact mid-point

$$
c_{N}(x)=\left(\frac{1-v_{1}^{2}}{h_{N}\left(k, v_{1}\right) E_{01}}+\frac{1-v_{2}^{2}}{h_{N}\left(k, v_{2}\right) E_{02}}\right)^{-1}\left(\frac{|x|}{c_{0}}\right)^{k} .
$$

The coefficient $h_{N}$ is dependent on the Poisson's ratio $v$ and the exponent $k$ of the inhomogeneity in the following way:

$$
h_{N}(k, v)=\frac{2(1+k) \cos \left(\frac{k \pi}{2}\right) \Gamma\left(1+\frac{k}{2}\right)}{\sqrt{\pi} C(k, v) \beta(k, v) \sin \left(\frac{\beta(k, v) \pi}{2}\right) \Gamma\left(\frac{1+k}{2}\right)},
$$

with

$$
C(k, v)=\frac{2^{1+k} \Gamma\left(\frac{3+k+\beta(k, v)}{2}\right) \Gamma\left(\frac{3+k-\beta(k, v)}{2}\right)}{\pi \Gamma(2+k)}
$$


and

$$
\beta(k, v)=\sqrt{(1+k)\left(1-\frac{k v}{1-v}\right)} .
$$

Thereby making use of the Gamma function:

$$
\Gamma(z):=\int_{0}^{\infty} t^{z-1} \exp (-t) \mathrm{d} t .
$$

Due to the mutual independence of the springs, the vertical displacement of the springs at location $x$ is given by the obvious equation

$$
w_{1 D}(x)=d-g(x)
$$

The indentation depth $d$ is determined from the condition of a vanishing displacement at the edge of the contact:

$$
w_{1 D}(a)=0 \quad \Rightarrow \quad d=g(a)
$$

The normal force $F_{N}$ is the sum of all spring forces in the contact:

$$
F_{N}(a)=\int_{-a}^{a} c_{N}(x) w_{1 D}(x) \mathrm{d} x
$$

Additionally, the normal displacement of the Winkler foundation $w_{1 D}(x)=d-g(x)$ uniquely defines the pressure distribution and the normal surface displacement:

$$
\begin{aligned}
& p(r ; a)=-\frac{c_{N}\left(c_{0}\right)}{\pi c_{0}^{k}} \int_{r}^{\infty} \frac{w_{1 D}^{\prime}(x)}{\left(x^{2}-r^{2}\right)^{\frac{1-k}{2}}} \mathrm{~d} x, \\
& w(r ; a)= \begin{cases}\frac{2 \cos \left(\frac{k \pi}{2}\right)}{\pi} \int_{0}^{r} \frac{x^{k} w_{1 D}(x)}{\left(r^{2}-x^{2}\right)^{\frac{1+k}{2}} \mathrm{~d} x} & \text { for } r \leq a, \\
\frac{2 \cos \left(\frac{k \pi}{2}\right)}{\pi} \int_{0}^{a} \frac{x^{k} w_{1 D}(x)}{\left(r^{2}-x^{2}\right)^{\frac{1+k}{2}}} \mathrm{~d} x & \text { for } r>a .\end{cases}
\end{aligned}
$$


These equations also solve sets of problems related to situations where the stresses at the half-space surface are known and the displacements are the desired quantities. This requires the preliminary calculation of the displacement of the Winkler foundation using the stresses according to

$$
w_{1 D}(x, a)=\frac{2 c_{0}^{k} \cos \left(\frac{k \pi}{2}\right)}{c_{N}\left(c_{0}\right)} \int_{x}^{a} \frac{r p(r)}{\left(r^{2}-x^{2}\right)^{\frac{1+k}{2}}} \mathrm{~d} r
$$

which are then inserted into (9.12).

Here it should be noted that contact problems between FGMs with arbitrary elastic inhomogeneities can always be represented by the MDR-based model from Fig. 9.2. The difficulty lies in finding the correct calculation formula for the onedimensional profile and determining the foundation modulus depending on the coordinate $x$ (Argatov et al. 2018). Taking advantage of the expanded model from Fig. 9.20, these statements even extend to adhesive contact problems.

\subsubsection{The Cylindrical Flat Punch}

The complete solution of the contact problem between a rigid, cylindrical flat punch and an inhomogeneous half-space (shown in Fig. 9.3) is attributed to Booker et al. (1985). The simple geometry of the contact allows for an extremely simple derivation of the solution using the MDR. Since the profile function is always measured from the indenter tip $f(r)=0$, it follows from (9.2) that $g(x)=0$. The surface displacement of the $1 \mathrm{D}$ Winkler foundation is then

$$
w_{1 D}(x)=d[\mathrm{H}(x+a)-\mathrm{H}(x-a)],
$$

where $\mathrm{H}(\cdot)$ represents the Heaviside function. For the contact of a flat punch, the indentation depth is independent of the (fixed) contact radius. Thus the evaluation

Fig. 9.3 Normal indentation of the elastically inhomogeneous half-space by a flat cylindrical punch

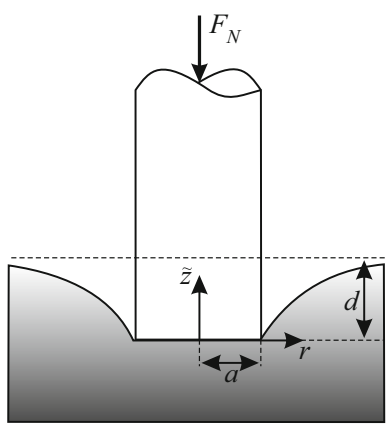


of (9.10) is not necessary and the solution of the contact problem merely requires applying (9.11) and (9.12). Under consideration of the derivative

$$
w_{1 D}^{\prime}(x)=d[\delta(x+a)-\delta(x-a)],
$$

with the Delta distribution $\delta(\cdot)$ and its filter property, it follows that:

$$
\begin{aligned}
F_{N}(d) & =\frac{2 h_{N}(k, v) E_{0} d a^{1+k}}{\left(1-v^{2}\right)(1+k) c_{0}^{k}}, \\
p(r ; d) & =-\frac{h_{N}(k, v) E_{0}}{\pi\left(1-v^{2}\right) c_{0}^{k}} \int_{r}^{\infty} \frac{d[\delta(x+a)-\delta(x-a)]}{\left(x^{2}-r^{2}\right)^{\frac{1-k}{2}}} \mathrm{~d} x \\
& =\frac{h_{N}(k, v) E_{0} d}{\pi\left(1-v^{2}\right) c_{0}^{k}\left(a^{2}-r^{2}\right)^{\frac{1-k}{2}}} \\
w(r ; d) & =\frac{2 \cos \left(\frac{k \pi}{2}\right)}{\pi} \int_{0}^{a} \frac{x^{k} d}{\left(r^{2}-x^{2}\right)^{\frac{1+k}{2}}} \mathrm{~d} x \\
& =\frac{\cos \left(\frac{k \pi}{2}\right) d}{\pi} \mathrm{B}\left(\frac{a^{2}}{r^{2}} ; \frac{1+k}{2}, \frac{1-k}{2}\right),
\end{aligned}
$$

where $\mathrm{B}(z ; x, y)$ represents the incomplete Beta function according to:

$$
\mathrm{B}(z ; x, y):=\int_{0}^{z} t^{x-1}(1-t)^{y-1} \mathrm{~d} t \quad \forall x, y \in \mathbb{R}^{+} .
$$

The pressure distribution normalized to the average pressure in the contact area is shown in Fig. 9.4. It is clear to see that a rising exponent of the elastic inhomo-

Fig. 9.4 Pressure distribution for the indentation by a flat cylindrical punch for different exponents of the elastic inhomogeneity $k$, normalized to the average pressure

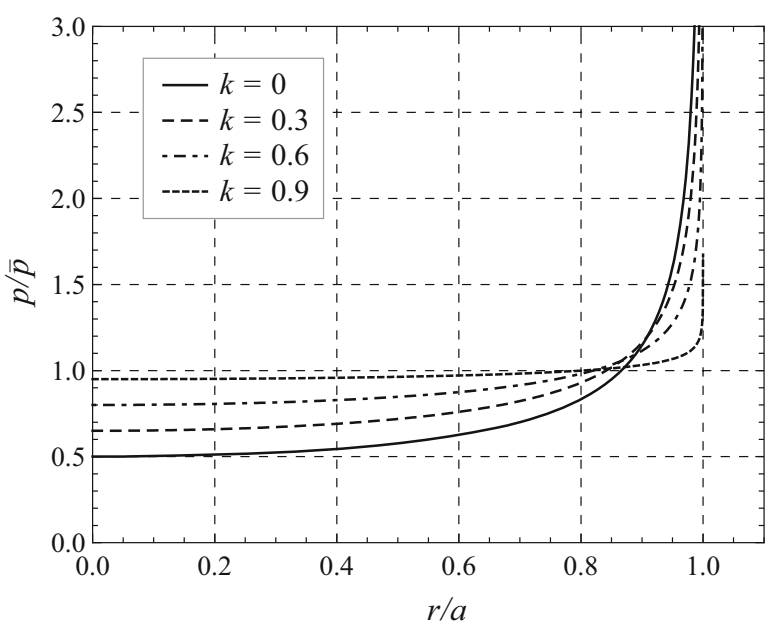




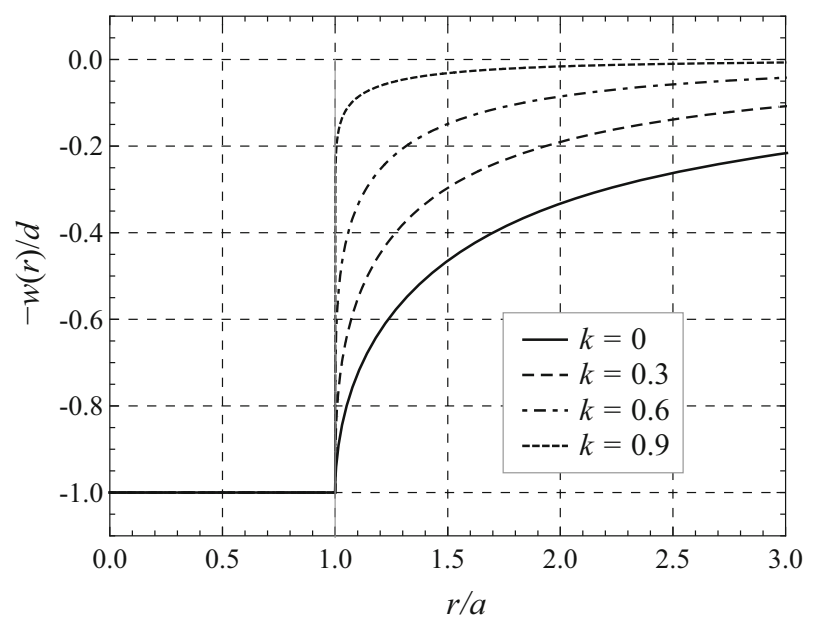

Fig. 9.5 Normal surface displacement of the inhomogeneous half-space for the contact of a flat punch and different exponents of the elastic inhomogeneity $k$, normalized to the indentation depth

geneity reduces the singularity at the contact edge. Figure 9.5 displays the normal surface displacements for different $k$ at equal indentation depth $d$. It shows that the displacement of the half-space surface outside the contact area drops with increasing $k$.

\subsubsection{The Cone}

Most solutions and the insights derived therefrom for the conical contact as shown in Fig. 9.6 can be found in the work of Giannakopoulos and Suresh (1997). The shape of the rigid conical indenter is given by:

$$
f(r)=r \tan \theta .
$$

Fig. 9.6 Normal indentation of the elastically inhomogeneous half-space by a conical indenter

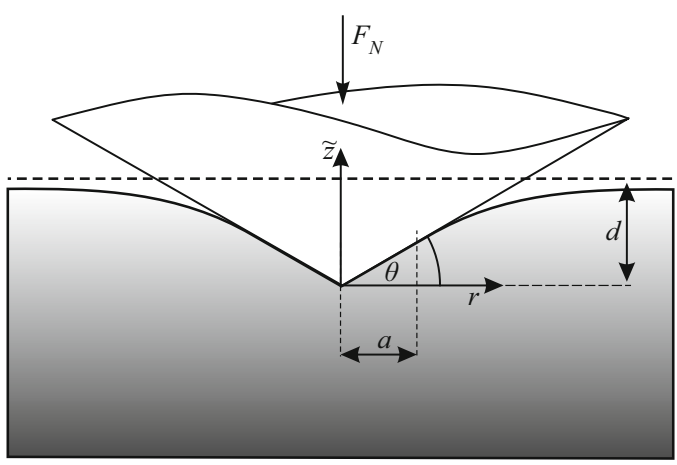


Applying the mapping rule (9.2) leads to the equivalent plane profile

$$
g(x)=\frac{1}{2}|x| \mathrm{B}\left(\frac{1}{2}, \frac{1+k}{2}\right) \tan \theta,
$$

where $\mathrm{B}(x, y)$ denotes the complete beta function, which is related to the incomplete beta function (9.17), according to $\mathrm{B}(x, y):=\mathrm{B}(1 ; x, y)$. Substituting (9.19) into (9.10)-(9.12), gives the solution of the contact problem, after a short calculation:

$$
\begin{aligned}
d(a)= & \frac{1}{2} a \tan \theta \mathrm{B}\left(\frac{1}{2}, \frac{1+k}{2}\right), \\
F_{N}(a)= & \frac{h_{N}(k, v) \tan \theta \mathrm{B}\left(\frac{1}{2}, \frac{1+k}{2}\right) E_{0} a^{k+2}}{\left(1-v^{2}\right) c_{0}^{k}(k+1)(k+2)}, \\
p(r ; a)= & \frac{h_{N}(k, v) \tan \theta \mathrm{B}\left(\frac{1}{2}, \frac{1+k}{2}\right) E_{0}}{4 \pi\left(1-v^{2}\right) c_{0}^{k}} r^{k} \\
& \cdot\left[\mathrm{B}\left(\frac{-k}{2}, \frac{1+k}{2}\right)-\mathrm{B}\left(\frac{r^{2}}{a^{2}} ; \frac{-k}{2}, \frac{1+k}{2}\right)\right], \\
w(r ; a)= & \frac{\tan \theta \mathrm{B}\left(\frac{1}{2}, \frac{1+k}{2}\right) \cos \left(\frac{k \pi}{2}\right) a}{2 \pi} \\
& \cdot\left[\mathrm{B}\left(\frac{a^{2}}{r^{2}} ; \frac{1+k}{2}, \frac{1-k}{2}\right)-\frac{r}{a} \mathrm{~B}\left(\frac{a^{2}}{r^{2}} ; \frac{3+k}{2}, \frac{1-k}{2}\right)\right] \quad \text { for } r>a .
\end{aligned}
$$

Since the beta function in the pressure distribution partially contains negative arguments, we expand the definition of (9.17) by using (for negative arguments) the representation via the hypergeometric series

$$
\mathrm{B}(z ; x, y)=\frac{z^{x}}{x}{ }_{2} \mathrm{~F}_{1}(x, 1-y ; 1+x ; z) .
$$

The definition of the hypergeometric series is given in Chap. 11 by (11.93). To obtain the limiting case of the homogeneous half-space, $k \rightarrow 0$ should be set.

From (9.20) it can be seen that (among other things) with the same normal force the contact radius depends both on the exponent $k$ and on the characteristic depth $c_{0}$. If one normalizes the contact radius by the contact radius $a_{h}$, which would be valid for contact with a homogeneous half-space, then it is

$$
\frac{a}{a_{h}}=\left(\frac{\pi(1+k)(2+k)}{2 h_{N}(k, v) \mathrm{B}\left(\frac{1}{2}, \frac{1+k}{2}\right)}\right)^{\frac{1}{2+k}}\left(\frac{c_{0}}{a_{h}}\right)^{\frac{k}{2+k}} .
$$

Depending on the choice of $k$ and $c_{0}$, a larger or smaller contact radius can result in comparison to the homogeneous half-space. This can be seen from the abscissa in Figs. 9.7 and 9.8 which illustrate the pressure distribution for various characteristic 
Fig. 9.7 Stress distribution in a contact with a cone for different exponents of elastic inhomogeneity $k$, normalized to the mean pressure $\bar{p}_{h}$, which results from contact with the homogeneous halfspace. The characteristic depth is $c_{0}=a_{h}$

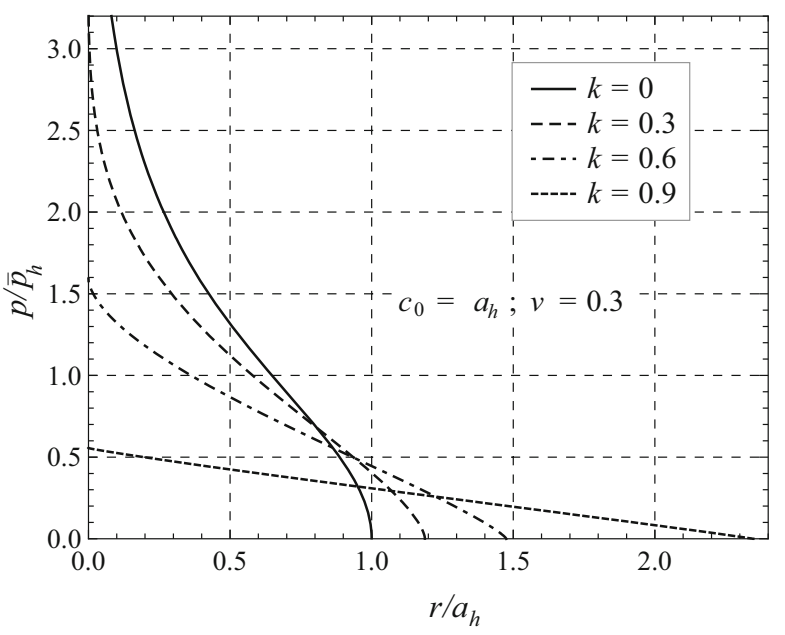

Fig. 9.8 Stress distribution in a contact with a cone for different exponents of elastic inhomogeneity $k$, normalized to the mean pressure $\bar{p}_{h}$, which results from contact with the homogeneous halfspace. The characteristic depth is $c_{0}=0.1 a_{h}$

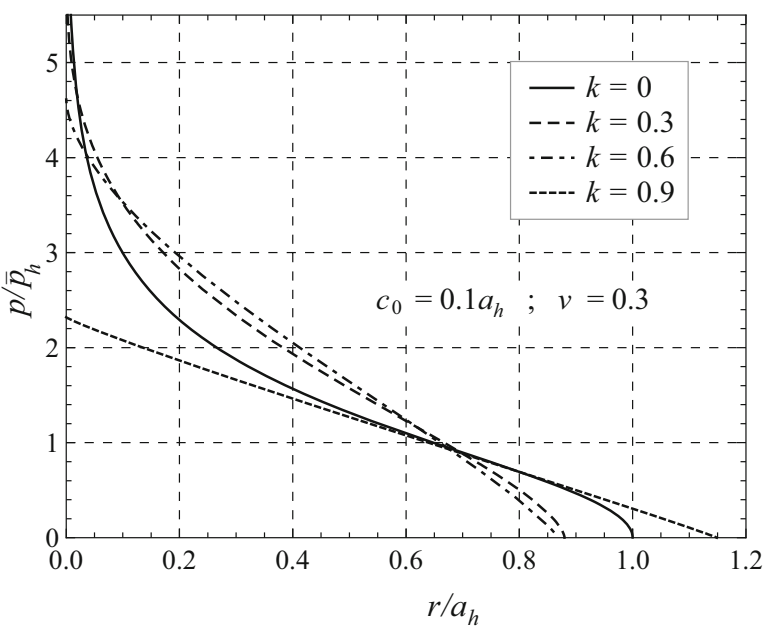

depths. Thereby the pressure was normalized to the average pressure $\bar{p}_{h}$ in conical contact with a homogeneous half-space, which is known to be independent of the contact radius:

$$
\bar{p}_{h}:=\frac{F_{N}}{\pi a_{h}^{2}}=\frac{E_{0} \tan \theta}{2\left(1-v^{2}\right)} .
$$

The influence of the elastic inhomogeneity is reflected, above all, in the maximum pressure in the center of the contact area. Because the pressure singularity in the homogeneous case is suppressed the pressure maximum takes a finite value.

In Fig. 9.9, the surface normal displacements of the inhomogeneous half-space are graphically compared for different exponents $k$ at the same contact radii. They were normalized to the indentation depth $d_{h}$, which results in contact with a homo- 


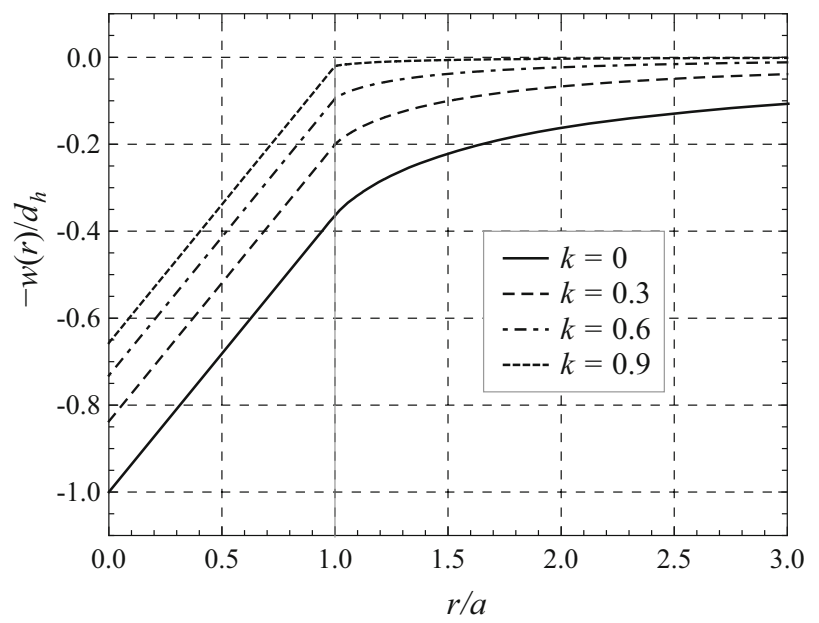

Fig. 9.9 Normalized normal displacements of the surface of the inhomogeneous half-space for a contact with a cone for different exponents of elastic inhomogeneity $k ; d_{h}$ is the indentation depth, corresponding to the homogeneous half-space

geneous half-space. By increasing $k$ the indentation depth and the displacements decrease successively.

\subsubsection{The Paraboloid}

Figure 9.10 shows the normal contact between a rigid parabolic indenter and an elastically inhomogeneous half-space. The shape of the rigid indenter is given by the function

$$
f(r)=\frac{r^{2}}{2 R}
$$

Fig. 9.10 Normal indentation of the elastically inhomogeneous half-space by a parabolic indenter

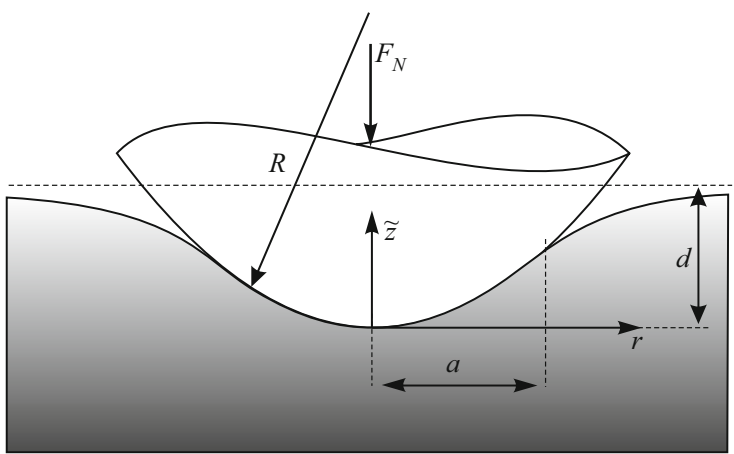


and can be understood as a (parabolic) approximation for a spherical contact with the radius of curvature $R$. The equivalent planar profile follows from (9.2):

$$
g(x)=\frac{x^{2}}{(k+1) R} .
$$

Considering (9.25), the evaluation of (9.10)-(9.12) provides the solution to the contact problem:

$$
\begin{aligned}
d(a) & =\frac{a^{2}}{(k+1) R}, \\
F_{N}(a) & =\frac{4 h_{N}(k, v) E_{0} a^{k+3}}{\left(1-v^{2}\right) c_{0}^{k}(k+1)^{2}(k+3) R}, \\
p(r ; a) & =\frac{2 h_{N}(k, v) E_{0} a^{k+1}}{\pi\left(1-v^{2}\right) c_{0}^{k}(k+1)^{2} R}\left[1-\left(\frac{r}{a}\right)^{2}\right]^{\frac{1+k}{2}}, \\
w(r ; a) & =\frac{a^{2} \cos \left(\frac{k \pi}{2}\right)}{(k+1) \pi R}\left[\mathrm{~B}\left(\frac{a^{2}}{r^{2}} ; \frac{1+k}{2}, \frac{1-k}{2}\right)\right. \\
& \left.-\frac{r^{2}}{a^{2}} \mathrm{~B}\left(\frac{a^{2}}{r^{2}} ; \frac{3+k}{2}, \frac{1-k}{2}\right)\right] \text { for } r>a,
\end{aligned}
$$

where $\mathrm{B}(z ; x, y)$ is the incomplete beta function from (9.17).

Figure 9.11 shows the pressure distribution in the parabolic contact normalized to the maximum pressure in the Hertzian contact. At the same normal force, a Poisson's number of $v=0.3$ and a characteristic depth $c_{0}$ (which was chosen equal to

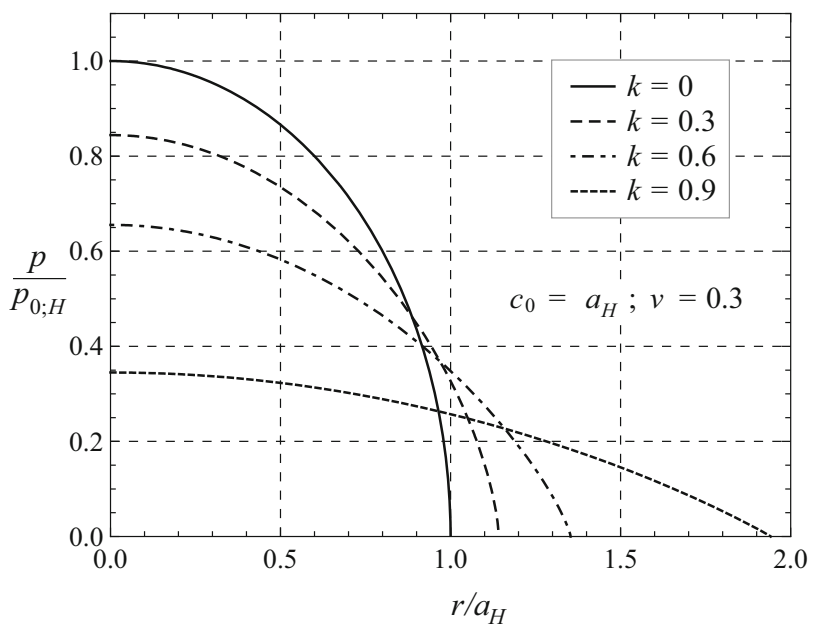

Fig. 9.11 Pressure distribution in parabolic contact for different exponents of elastic inhomogeneity $k$, normalized to the maximum pressure of Hertzian contact $p_{0, H}$ 


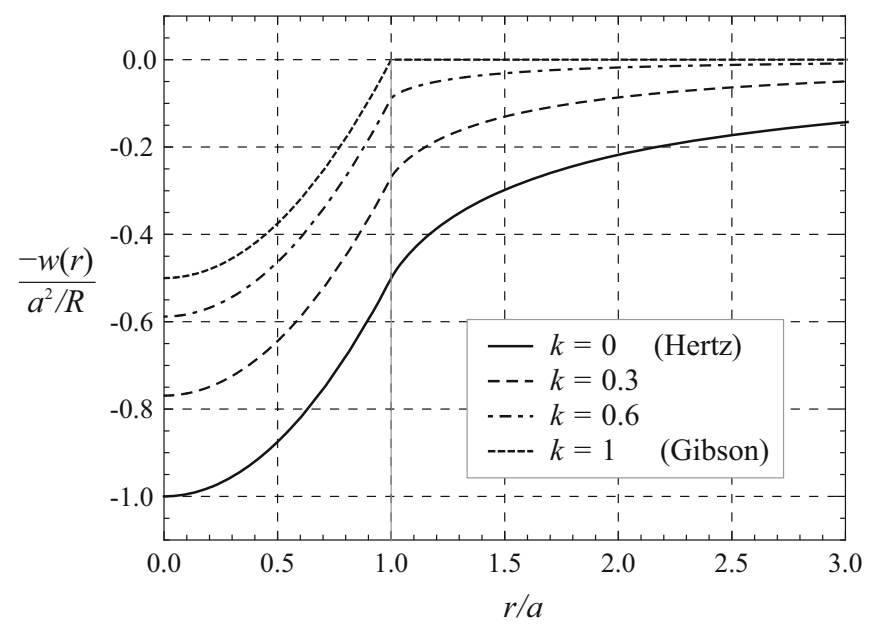

Fig. 9.12 Normalized surface normal displacements of the inhomogeneous half-space in a parabolic contact and different exponents of elastic inhomogeneity $k$

the contact radius $a_{H}$ in Hertzian contact), the maximum pressure decreases with increasing exponent $k$ of the elastic inhomogeneity with simultaneous enlargement of the contact area. It should be noted that, depending on the choice of the characteristic depth and the Poisson's number, an opposite effect can also occur. In Fig. 9.12, the normalized normal displacements of the surface of the inhomogeneous half-space are visualized for different exponents $k$ at the same contact radii. By increasing $k$ the indentation depth and the displacements decrease successively.

In the limiting case $k=1$ the displacements outside the contact area disappear completely - the inhomogeneous half-space behaves like a (two-dimensional) Winkler foundation. Strictly speaking, the latter behavior is coupled to the linearinhomogeneous incompressible half-space because only the $v=0.5$ contact has a non-zero contact stiffness (see Sect. 9.1.8).

\subsubsection{The Profile in the Form of a Power-Law}

The contact between the inhomogeneous half-space and an axisymmetric indenter whose shape meets the power-law

$$
f(r)=A_{n} r^{n} \quad \text { with } n \in \mathbb{R}^{+}, A_{n}=\text { const }
$$

(see Fig. 9.13) was examined by Rostovtsev (1961) for the special case $v=1 /(2+k)$. A general solution was provided by Giannakopoulos and Suresh (1997). Their derivation by means of the MDR requires the calculation of the equivalent plane profile according to (9.2):

$$
g(x)=\kappa(n, k) A_{n}|x|^{n}=\kappa(n, k) f(|x|) .
$$


Fig. 9.13 Normal indentation of the elastically inhomogeneous half-space by an indenter whose profile is described by a power function

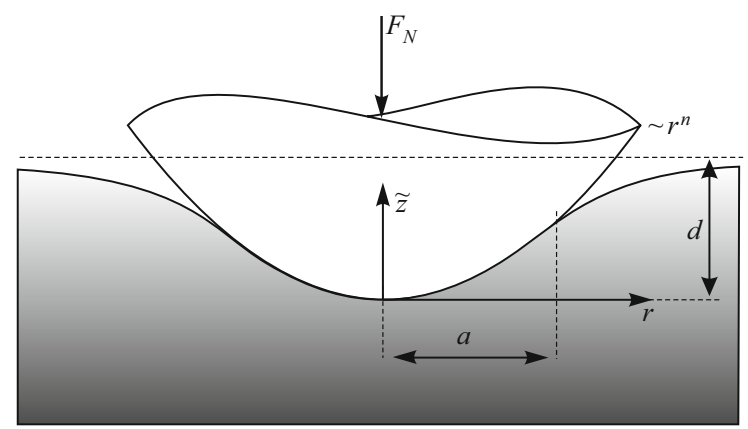

Equation (9.28) clearly shows that the equivalent profile results from a simple stretch from the original profile. The stretch factor is dependent on the exponent of the power function and the exponent of the elastic inhomogeneity,

$$
\begin{aligned}
\kappa(n, k) & =\int_{0}^{1} \frac{n \varsigma^{n-1}}{\left(1-\varsigma^{2}\right)^{\frac{1-k}{2}}} \mathrm{~d} \zeta=\frac{n}{2} \int_{0}^{1} t^{\frac{n}{2}-1}(1-t)^{\frac{k+1}{2}-1} \mathrm{~d} t \\
& =: \frac{n}{2} \mathrm{~B}\left(\frac{n}{2}, \frac{k+1}{2}\right),
\end{aligned}
$$

where $\mathrm{B}(x, y)$ denotes the complete beta function, which follows as a special case $\mathrm{B}(x, y):=\mathrm{B}(1 ; x, y)$ from the definition of the incomplete beta function (9.17).

The stretch factor increases as the exponent of the power profile increases (see Fig. 9.14). In homogeneous cases, the known values $\kappa(1,0)=\pi / 2$ for the conical

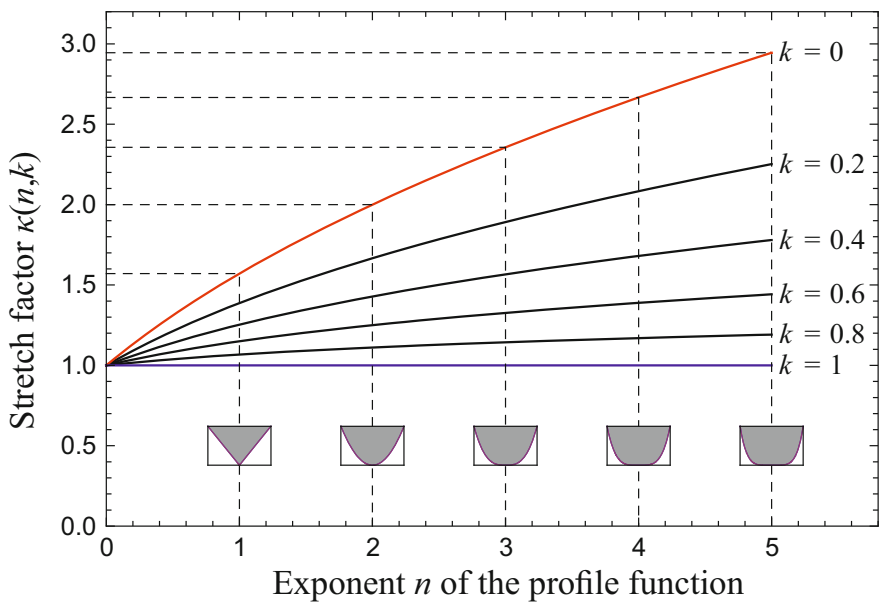

Fig. 9.14 Dependence of the stretch factor $\kappa$ on the exponent $n$ of the power-law profile function for different exponents $k$ of the elastic inhomogeneity (from Heß 2016a) 
and $\kappa(2,0)=2$ for the parabolic indenter are reproduced. As the exponent of elastic inhomogeneity increases, the stretch factor decreases. The limiting case, $k \rightarrow 1$ coincides with the simple cross-section of the three-dimensional profile in the $x-z$-plane. lem:

The application of rules (9.10)-(9.12) leads to the solution of the contact prob-

$$
\begin{aligned}
d(a)= & \kappa(n, k) A_{n} a^{n}, \\
F_{N}(a)= & \frac{2 h_{N}(k, v) \kappa(n, k) n A_{n} E_{0} a^{n+k+1}}{\left(1-v^{2}\right) c_{0}^{k}(k+1)(n+k+1)}, \\
p(r ; a)= & \frac{h_{N}(k, v) \kappa(n, k) n A_{n} E_{0}}{2 \pi\left(1-v^{2}\right) c_{0}^{k}} r^{n+k-1} \\
& \cdot\left[\mathrm{B}\left(\frac{1-k-n}{2}, \frac{1+k}{2}\right)-\mathrm{B}\left(\frac{r^{2}}{a^{2}} ; \frac{1-k-n}{2}, \frac{1+k}{2}\right)\right], \\
w(r ; a)= & \frac{\cos \left(\frac{k \pi}{2}\right) \kappa(n, k) A_{n} a^{n}}{\pi} \\
& \cdot\left[\mathrm{B}\left(\frac{a^{2}}{r^{2}} ; \frac{1+k}{2}, \frac{1-k}{2}\right)\right. \\
& \left.-\left(\frac{r}{a}\right)^{n} \mathrm{~B}\left(\frac{a^{2}}{r^{2}} ; \frac{1+k+n}{2}, \frac{1-k}{2}\right)\right] \text { for } r>a .
\end{aligned}
$$

As already discussed in the context of the investigation of the conical contact (Sect. 9.1.3), the expanded definition of the beta function according to (9.21) should be used.

\subsubsection{The Concave Paraboloid (Complete Contact)}

The shape of a cylindrical indenter with a parabolic-concave end is described by:

$$
f(r)=-h_{0} \frac{r^{2}}{a^{2}} \quad \text { for } 0 \leq r \leq a \quad \text { with } h_{0}=\frac{a^{2}}{2 R} .
$$

The complete indentation of such a punch (so that all points of the face are in contact) leads to a surface displacement in the contact area

$$
w\left(r ; d_{0}\right)=d_{0}-f(r)
$$

where $d_{0}$ denotes the displacement in the center of the punch. The contact geometry is shown in Fig. 9.15. Adding a suitable rigid body displacement fraction and taking 
Fig. 9.15 Normal indentation of the elastically inhomogeneous half-space by a parabolic concave indenter

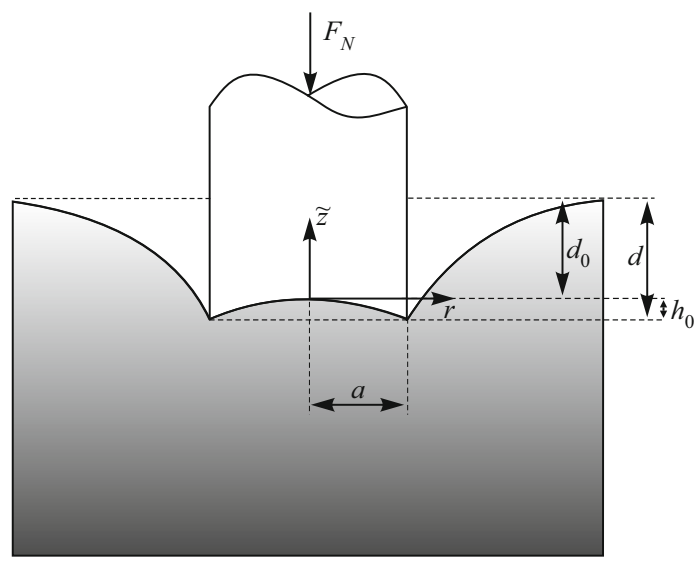

into account (9.31), (9.32) can be written as follows:

$$
w\left(r ; d_{0}\right)=\underbrace{d_{0}+\frac{a^{2}}{(k+1) R}}_{=w_{1}}-\underbrace{\left(\frac{a^{2}}{(k+1) R}-\frac{r^{2}}{2 R}\right)}_{=w_{2}} .
$$

According to (9.33), the displacement can be represented as the difference between the rigid body displacement $w_{1}$ and the displacement $w_{2}$ resulting from a parabolic contact. For both separate fractions the solutions have already been developed (see (9.16) and (9.26)). Due to the validity of the superposition principle, the overall solution is the difference of the partial solutions

$$
\begin{aligned}
F_{N}\left(d_{0}\right)= & F_{1}-F_{2}=\frac{2 h_{N}(k, v) E_{0} a^{k+1}}{\left(1-v^{2}\right)(1+k) c_{0}^{k}}\left(d_{0}+\frac{2 h_{0}}{k+3}\right), \\
p\left(r ; d_{0}\right)= & p_{1}-p_{2} \\
= & \frac{h_{N}(k, v) E_{0}}{\pi\left(1-v^{2}\right) c_{0}^{k}\left(a^{2}-r^{2}\right)^{\frac{1-k}{2}}}\left[d_{0}+\frac{2 h_{0}}{(k+1)^{2}}\left(k-1+2 \frac{r^{2}}{a^{2}}\right)\right], \\
w\left(r ; d_{0}\right)= & w_{1}-w_{2} \\
= & \frac{\cos \left(\frac{k \pi}{2}\right)}{\pi}\left[d_{0} \mathrm{~B}\left(\frac{a^{2}}{r^{2}} ; \frac{1+k}{2}, \frac{1-k}{2}\right)\right. \\
& \left.\quad+\frac{2 h_{0}}{k+1} \frac{r^{2}}{a^{2}} \mathrm{~B}\left(\frac{a^{2}}{r^{2}} ; \frac{3+k}{2}, \frac{1-k}{2}\right)\right] .
\end{aligned}
$$

With the exeption of the displacements, solutions (9.34) were derived by Jin et al. (2013). It should be emphasized that these solutions are only valid under the condition of complete contact; for this, the requirement $p(r=0)>0$ must be fulfilled, 


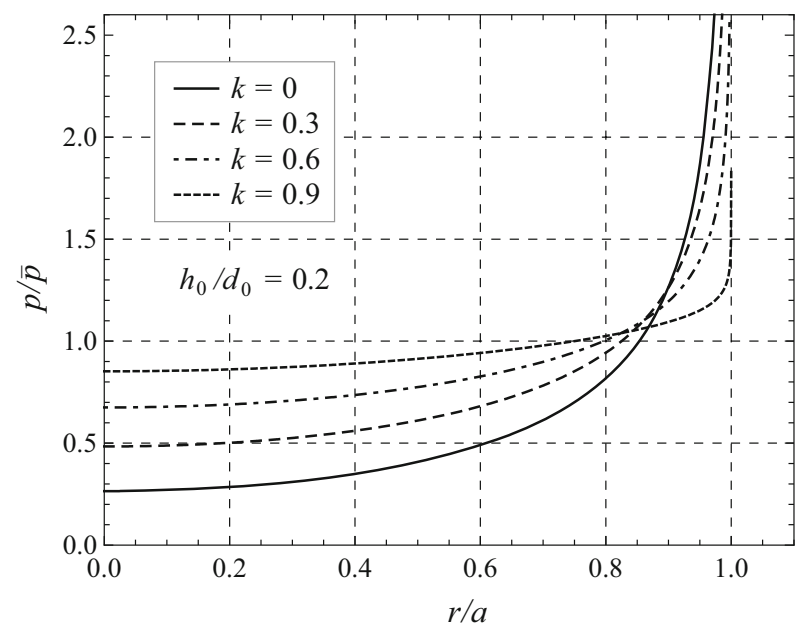

Fig. 9.16 Pressure distribution when indented by a cylindrical punch with parabolic-concave tip for different exponents of elastic inhomogeneity $k$ and $h_{0} / d_{0}=0.2$, normalized to the mean pressure $\bar{p}$ in the contact area

which suggests:

$$
d_{0}>\frac{2(1-k)}{(1+k)^{2}} h_{0} \quad \text { or } \quad F_{N}>\frac{16 h_{N}(k, v) E_{0} a^{1+k} h_{0}}{\left(1-v^{2}\right) c_{0}^{k}(1+k)^{3}(3+k)} .
$$

Figure 9.16 shows the pressure distribution in the contact area, normalized to the mean pressure $\bar{p}$ for $h_{0}=0.2 d_{0}$. As the $k$ increases the graph progressively approaches the shape of the indenter. For the same specification, the surface displacements are illustrated in Fig. 9.17.

Finally, to solve contact problems with concave profiles, the MDR rules (9.2), (9.11), and (9.12) are still valid. However, care must be taken to ensure that the center displacement $d_{0}$ is used instead of the indentation depth. In addition, complete contact in the replacement model does not necessarily result in full contact with the original problem so the requirement $p(r)>0$ always has to be checked. For the contact problem investigated here the following is valid:

$$
g(x)=-\frac{2 h_{0}}{k+1} \frac{x^{2}}{a^{2}} \Rightarrow w_{1 D}(x):=d_{0}-g(x)=d_{0}+\frac{2 h_{0}}{k+1} \frac{x^{2}}{a^{2}} .
$$

An evaluation of (9.11) and (9.12), and taking into account (9.36), gives solutions (9.34) exactly. 


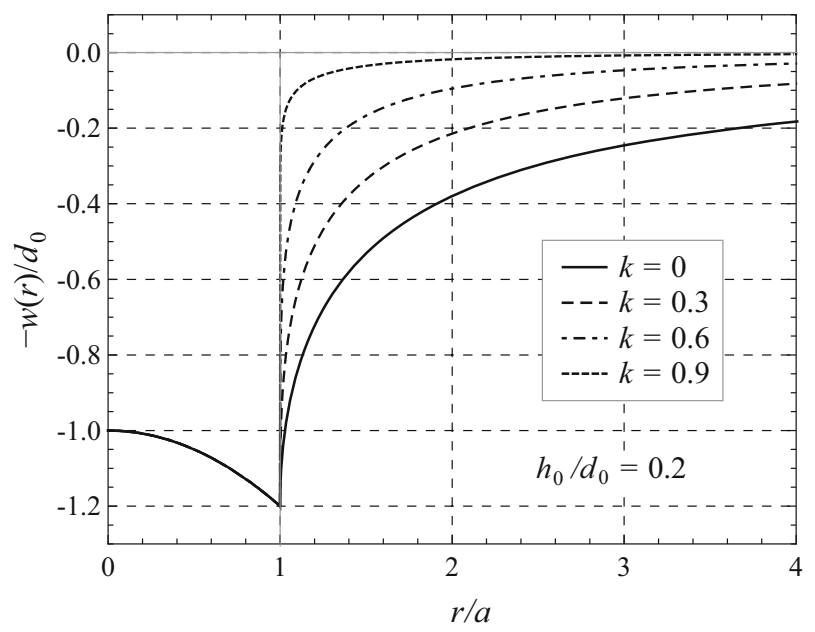

Fig. 9.17 Surface normal displacements normalized by the center displacement $d_{0}$ on impression with a cylindrical indenter with parabolic-concave tip for different exponents of elastic inhomogeneity $k$ and $h_{0} / d_{0}=0.2$

\subsubsection{The Profile That Generates Constant Pressure}

In contrast to the previous contact problems, where the indenter shape was known a priori, the issue now lies in determining the surface displacement caused by a known constant pressure distribution:

$$
p(r)=p_{0} \text { for } 0 \leq r \leq a .
$$

This requires first determining the 1D displacement of the foundation according to (9.13):

$$
\begin{aligned}
w_{1 D}(x) & =\frac{2 c_{0}^{k}\left(1-v^{2}\right) \cos \left(\frac{k \pi}{2}\right)}{h_{N}(k, v) E_{0}} \int_{x}^{a} \frac{r p_{0}}{\left(r^{2}-x^{2}\right)^{\frac{1+k}{2}}} \mathrm{~d} r \\
& =\frac{2 c_{0}^{k}\left(1-v^{2}\right) \cos \left(\frac{k \pi}{2}\right) p_{0}\left(a^{2}-x^{2}\right)^{\frac{1-k}{2}}}{h_{N}(k, v)(1-k) E_{0}} .
\end{aligned}
$$

The displacement at location $r=0$ in the original must coincide with the displacement at location $x=0$ in the substitute model, as follows:

$$
w_{c}:=w(r=0) \equiv w_{1 D}(x=0)=\frac{2 c_{0}^{k}\left(1-v^{2}\right) \cos \left(\frac{k \pi}{2}\right)}{h_{N}(k, v)(1-k) E_{0} \pi} \frac{F_{N}}{a^{1+k}},
$$

taking into account that $F_{N}=p_{0} \pi a^{2}$. When the stresses are specified instead of the indenter shape, the equivalent quantity to the indentation depth is the center displacement. Inserting (9.38) in (9.12) yields, after a short calculation, the following 


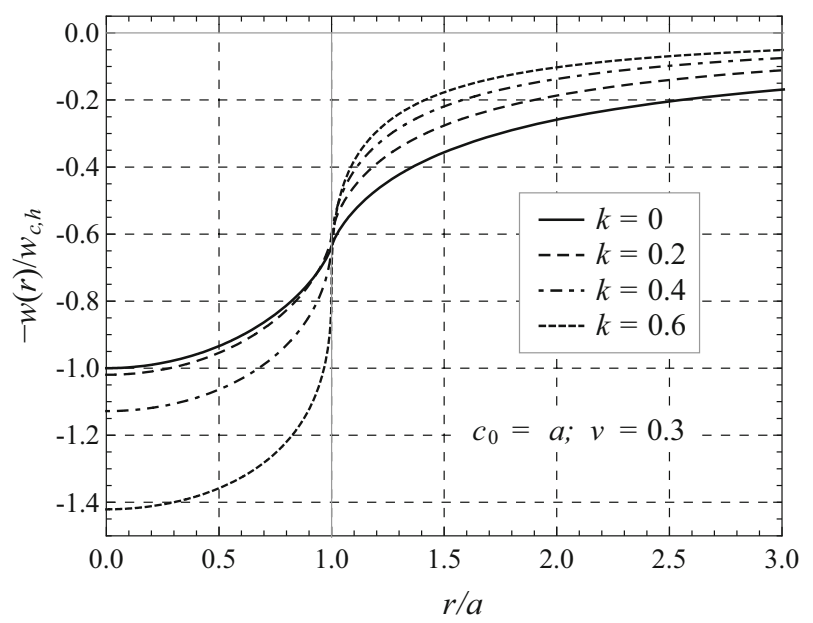

Fig. 9.18 Normalized normal surface displacement of the inhomogeneous half-space for a constant pressure distribution and for different exponents of the elastic inhomogeneity $k$ and fixed $c_{0}$

surface displacements of the inhomogeneous half-space:

$$
w(r ; a)= \begin{cases}w_{c 2} \mathrm{~F}_{1}\left(\frac{k-1}{2}, \frac{1+k}{2} ; 1 ; \frac{r^{2}}{a^{2}}\right) & \text { for } r \leq a, \\ w_{c} \frac{1-k}{2}\left(\frac{a}{r}\right){ }_{2}^{1+k} \mathrm{~F}_{1}\left(\frac{1+k}{2}, \frac{1+k}{2} ; 2 ; \frac{a^{2}}{r^{2}}\right) & \text { for } r>a,\end{cases}
$$

which coincide with ones calculated by Booker et al. (1985). The displacements normalized to the center displacement of the homogeneous half-space,

$$
w_{c, h}=\frac{2\left(1-v^{2}\right) p_{0} a}{E_{0}},
$$

are displayed in Figs. 9.18 and 9.19. Fig. 9.18 studies the influence of the elastic inhomogeneity exponent at a fixed characteristic depth $c_{0}$. For the chosen characteristic depth, an increasing exponent $k$ causes a rise of the displacements within the load zone and a drop outside this zone. We consciously avoided particularly large exponents $k$ since it results in unbounded displacements within the load zone (see the discussion in Sect. 9.1.8). Figure 9.19, on the other hand, demonstrates decreasing displacements for a reduction of the characteristic depth, which is fixed at $k=0.2$. It should be noted that these graphs are offered as examples and do not present all characteristics. However, a complete analysis of the fundamental displacement behavior depending on $k, c_{0}$, and $p_{0}$ can already be gained from the power-law of the elastic inhomogeneity from (9.1). 


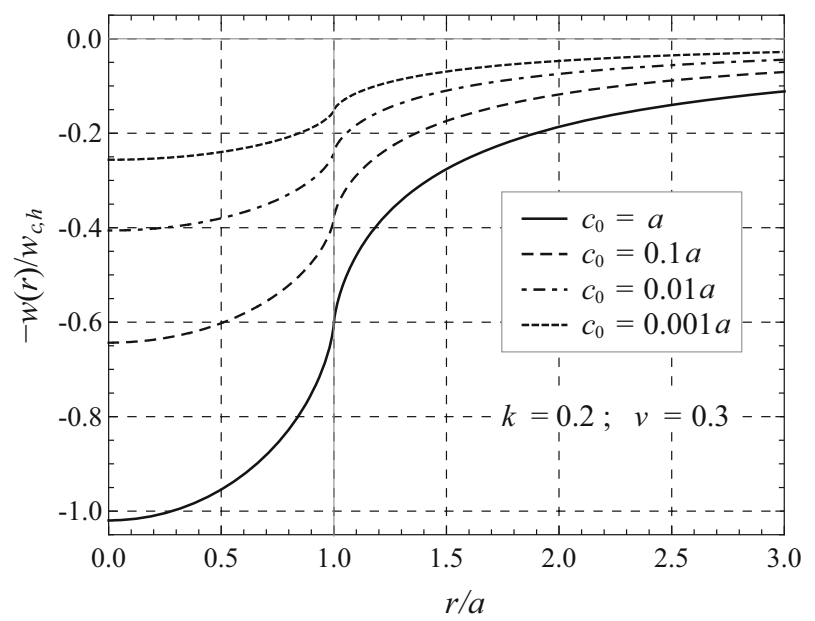

Fig. 9.19 Normalized normal surface displacement of the inhomogeneous half-space for a constant pressure distribution and for different characteristic depths $c_{0}$, and fixed exponent $k$ of the elastic inhomogeneity

\subsubsection{Notes on the Linear-Inhomogeneous Half-Space-the Gibson Medium}

In a linear-inhomogeneous half-space, the elasticity modulus increases proportionally with the depth $z$. This dependency is covered by the general power-law (9.1); it merely requires the setting of $k=1$. Accounting for this condition in (9.2)-(9.12) exposes some interesting characteristics, independent of the geometry of the contact. For instance, it follows that the equivalent planar profile coincides with the cross-section of the real profile in the $x$-z-plane,

$$
g(x)=f(|x|) .
$$

Hence the rule for the calculation of the indentation depth (9.10) already returns an unusual result of

$$
d=f(a) \quad \Rightarrow \quad w(r)=f(a)-f(r) \quad \text { for } 0 \leq r \leq a .
$$

According to (9.43), the displacement of the half-space surface at the contact edge is zero like in the 1D model. The calculation formula for the displacements of (9.12) even reveals that the half-space surface exterior of the contact area remains in its original, undeformed state. Such behavior is typical for a (two-dimensional) Winkler foundation, whose surface points are displaced in proportion to the normal stresses acting locally at these points. However, an evaluation of the rule for the calculation of the pressure distribution from (9.12) reveals that this proportionality is valid only for the special case of a linear-homogeneous, incompressible halfspace since the factor $h(1, v)$ takes on a non-zero value only for $v=0.5$. With 
$h(1,0.5)=\pi / 2$ it follows that:

$$
p(x, y)=\frac{2 E_{0}}{3 c_{0}} w(x, y) .
$$

The latter finding goes back to Gibson (1967), which is why the linear-inhomogeneous incompressible half-space is also named the Gibson medium. A normal load on a linear-inhomogeneous, compressible half-space results in unbounded surface displacements within the load zone (Awojobi and Gibson 1973). This is indicated by (9.11). The foundation modulus vanishes according to (9.4). Therefore the effects of an external force cannot be balanced out. The indeterminateness of the displacements is a consequence of the vanishing elasticity modulus at the half-space surface in a medium defined by (9.1) (Brown and Gibson 1972).

\subsection{Frictionless Normal Contact with JKR Adhesion}

\subsubsection{Basis for Calculation of the MDR and General Solution}

The framework provided by the JKR theory (see Sects. 3.2 and 3.3 in Chap. 3 of this book) permits a particularly easy solution to contact problems with adhesion since the basic idea relies on a simple superposition of the corresponding non-adhesive contact and a rigid-body translation. The latter does depend on the particular contact radius but not on the shape of the indenter. Application of this approach to contact problems related to the elastically inhomogeneous half-space gives the indentation depth as a function of the contact radius

$$
d(a)=d_{\text {n.a. }}(a)-\Delta \ell(a) \quad \text { with } \quad \Delta \ell(a):=\sqrt{\frac{2 \pi \Delta \gamma c_{0}^{k} a^{1-k}}{E^{*} h_{N}(k, v)}} .
$$

Here, $d_{\text {n.a. }}$ refers to the indentation depth of the contact without adhesion (which would lead to the same contact radius as the one of the adhesive contact). The unusual notation of the superimposed rigid-body translation $\Delta \ell(a)$ stems from the substitute model of the MDR displayed in Fig. 9.20.

Fig. 9.20 Equivalent substitute model for the adhesive normal contact between two elastically inhomogeneous half-spaces, whose elasticity moduli satisfy condition (9.1), assuming equal exponents $k$ of both media

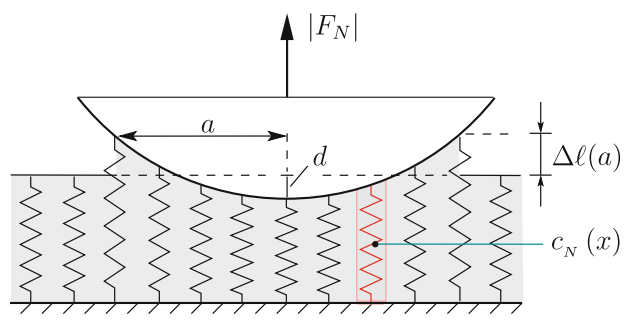


For the complete solution of a contact problem with adhesion via the MDR, all rules and calculation formulas from Sect. 9.1.1 remain valid with the exception of (9.10). It must be replaced by the condition

$$
w_{1 D}(a)=-\Delta \ell(a)=-\sqrt{\frac{2 \pi \Delta \gamma c_{0}^{k} a^{1-k}}{E^{*} h_{N}(k, v)}}
$$

with the displacement of the spring elements given by the same equation as in the non-adhesive case:

$$
w_{1 D}(x)=d-g(x) .
$$

This means that (under the condition that all springs in the contact area remain in contact) the critical detachment state of the edge springs is achieved when the elongation of the springs at the edge reaches the pre-defined value $\Delta \ell(a)$ (see Fig. 9.20).

For convex profiles, the solution approach via the MDR additionally offers a simple way to calculate the critical contact radii (after achieving this, no equilibrium state of the contact as a whole exists). The critical contact radii satisfies the following equation:

$$
\frac{\Delta \ell\left(a_{c}\right)}{a_{c}}=\left.\tilde{C}(k) \frac{\partial g(a)}{\partial a}\right|_{a_{c}} \quad \text { with } \tilde{C}(k):= \begin{cases}\frac{2}{3+k} & \text { for } F_{N}=\text { const } \\ \frac{2}{1-k} & \text { for } d=\text { const. }\end{cases}
$$

The definition of the coefficient $\tilde{C}(k)$ varies depending on whether the experiment is force-controlled or displacement-controlled (Heß 2016a).

Taking (9.47) into consideration, (9.11) and (9.12) yield the general solution of the contact with adhesion:

$$
\begin{aligned}
& F_{N}(a)=F_{\text {n.a. }}(a)-\frac{2 h_{N}(k, v) E^{*} \Delta \ell(a) a^{1+k}}{(1+k) c_{0}^{k}}, \\
& p(r ; a)=p_{\text {n.a. }}(r ; a)-\frac{h_{N}(k, v) E^{*} \Delta \ell(a)}{\pi c_{0}^{k}\left(a^{2}-r^{2}\right)^{\frac{1-k}{2}}}, \\
& w(r ; a)= \begin{cases}d_{\text {n.a. }}(a)-f(r)-\Delta \ell(a) & \text { for } r \leq a, \\
w_{\text {n.a. }}(r ; a)-\frac{\cos \left(\frac{k \pi}{2}\right) \Delta \ell(a)}{\pi} \mathrm{B}\left(\frac{a^{2}}{r^{2}} ; \frac{1+k}{2}, \frac{1-k}{2}\right) & \text { for } r>a .\end{cases}
\end{aligned}
$$

The quantities with the indices "n.a." indicate the solutions of the non-adhesive contact. The additional terms result from the rigid-body translation and correspond 
to the solutions of the flat punch contact (if, instead of the indentation depth $d$, the (negative) rigid-body translation $-\Delta \ell(a)$ is used).

\subsubsection{The Cylindrical Flat Punch}

When applying a tensile force to a cylindrical flat punch of radius $a$, which is adhering to the surface of the inhomogeneous half-space, all points in the contact area are subjected to the same displacement $w(r)=d<0$. Therefore, except for the negative value of the indentation depth $d$, the boundary conditions match those of the indentation of a cylindrical flat punch in an inhomogeneous half-space. Therefore, we can adopt the solutions from Sect. 9.1.2:

$$
\begin{aligned}
F_{N}(d) & =\frac{2 h_{N}(k, v) E_{0} d a^{1+k}}{\left(1-v^{2}\right)(1+k) c_{0}^{k}}, \\
p(r ; d) & =\frac{h_{N}(k, v) E_{0} d}{\pi\left(1-v^{2}\right) c_{0}^{k}\left(a^{2}-r^{2}\right)^{\frac{1-k}{2}}}, \\
w(r ; d) & =\frac{\cos \left(\frac{k \pi}{2}\right) d}{\pi} \mathrm{B}\left(\frac{a^{2}}{r^{2}} ; \frac{1+k}{2}, \frac{1-k}{2}\right) .
\end{aligned}
$$

Since the contact radius cannot (stably) decrease in a tensile test, the adhesive contact radius of the flat punch becomes unstable once the "indentation depth" reaches the critical value

$$
d_{c}(a)=-\Delta \ell(a)=-\sqrt{\frac{2 \pi \Delta \gamma c_{0}^{k} a^{1-k}}{E^{*} h_{N}(k, v)}} .
$$

In this case, the flat punch will instantly completely detach from the half-space surface. The corresponding normal force, the absolute value of which is the maximum pull-off force, is:

$$
F_{c}(a)=-\int_{-a}^{a} c_{N}(x) \Delta \ell(a) \mathrm{d} x=-\sqrt{\frac{8 \pi \Delta \gamma h_{N}(k, v) E^{*} a^{3+k}}{(k+1)^{2} c_{0}{ }^{k}}} .
$$

A comparison of (9.51) and (9.52) with the equilibrium conditions (9.49) for curved profiles reveals that the solution of the contact without adhesion is calculated by summing the aforementioned terms. This is a pre-requisite step for the solution of the adhesive contact.

\subsubsection{The Paraboloid}

The solution of the contact problem between a parabolic, rigid indenter and the elastically inhomogeneous half-space, displayed in Fig. 9.21, is gained from the 
Fig. 9.21 Adhesive normal contact between a rigid parabolic indenter and an elastically inhomogeneous half-space

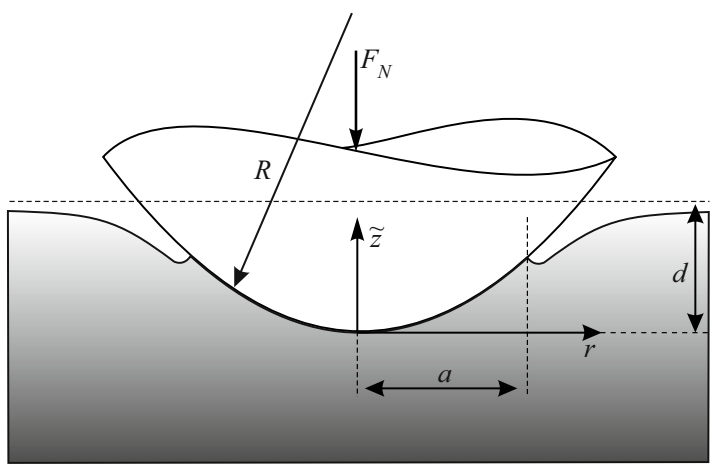

universal superposition (9.49) by incorporating the solution of the non-adhesive contact problem (9.26):

$$
\begin{aligned}
d(a)= & \frac{a^{2}}{(k+1) R}-\sqrt{\frac{2 \pi \Delta \gamma c_{0}^{k} a^{1-k}}{E^{*} h_{N}(k, v)}}, \\
F_{N}(a)= & \frac{4 h_{N}(k, v) E^{*} a^{k+3}}{c_{0}^{k}(k+1)^{2}(k+3) R}-\sqrt{\frac{8 \pi \Delta \gamma h_{N}(k, v) E^{*} a^{3+k}}{(k+1)^{2} c_{0} k}}, \\
p(r ; a)= & \frac{2 h_{N}(k, v) E^{*} a^{k+1}}{\pi c_{0}^{k}(k+1)^{2} R}\left[1-\left(\frac{r}{a}\right)^{2}\right]^{\frac{1+k}{2}} \\
& -\sqrt{\frac{2 h_{N}(k, v) \Delta \gamma E^{*} a^{k-1}}{\pi c_{0} k}}\left[1-\left(\frac{r}{a}\right)^{2}\right]^{\frac{k-1}{2}}, \\
w(r ; a)= & \frac{a^{2} \cos \left(\frac{k \pi}{2}\right)}{(k+1) \pi R}\left[\left(1-(1+k) R \sqrt{\left.\frac{2 \pi \Delta \gamma c_{0}^{k} a^{-3-k}}{E^{*} h_{N}(k, v)}\right)}\right.\right. \\
\cdot & \left.\mathrm{B}\left(\frac{a^{2}}{r^{2}} ; \frac{1+k}{2}, \frac{1-k}{2}\right)-\frac{r^{2}}{a^{2}} \mathrm{~B}\left(\frac{a^{2}}{r^{2}} ; \frac{3+k}{2}, \frac{1-k}{2}\right)\right] .
\end{aligned}
$$

The calculation of the critical contact radii from the condition of global stability (9.48) merely requires a knowledge of the slope of the equivalent substitute profile at the contact edge. From (9.25) it follows that

$$
g^{\prime}(a)=\frac{2 a}{(1+k) R},
$$

and thus, with (9.48), a short calculation yields:

$$
a_{c}=\left(\frac{\pi(1+k)^{2} R^{2} \Delta \gamma c_{0}{ }^{k}}{2 \tilde{C}(k)^{2} h_{N}(k, v) E^{*}}\right)^{\frac{1}{3+k}} .
$$




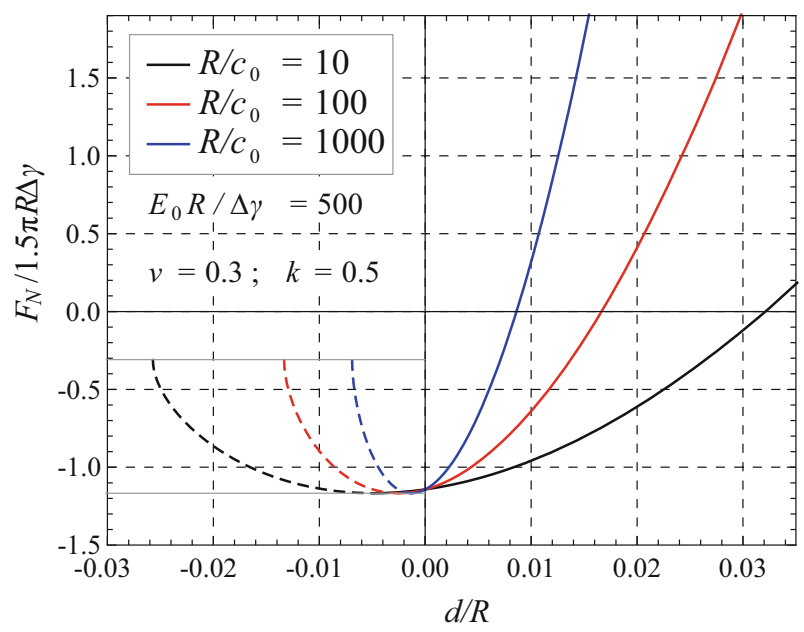

Fig. 9.22 Normal force as a function of the indentation depth in normalized representation for $k=0.5$ at different characteristic depths $c_{0}$

Inserting the critical contact radii (9.55) into the first two equations of (9.53) returns the following critical indentation depths and normal forces:

$$
\begin{aligned}
d_{c} & =\frac{1-2 \tilde{C}(k)}{(1+k) R}\left(\frac{\pi(1+k)^{2} R^{2} \Delta \gamma c_{0}^{k}}{2 \tilde{C}(k)^{2} h_{N}(k, v) E^{*}}\right)^{\frac{2}{3+k}}, \\
F_{c} & =\left(\frac{1-\tilde{C}(k)(3+k)}{\tilde{C}(k)^{2}(3+k)}\right) 2 \pi \Delta \gamma R \\
& = \begin{cases}-\frac{k+3}{2} \pi \Delta \gamma R & \text { (force-control) } \\
-\frac{(1-k)(5+3 k)}{2(3+k)} \pi \Delta \gamma R & \text { (displacement-control). }\end{cases}
\end{aligned}
$$

Solutions (9.53)-(9.56) were derived by Chen et al. (2009), and are focused on force-controlled experiments. They noted that, according to (9.56), the maximum pull-off force (absolute value of the critical normal force under force-control) is independent of the elasticity parameters and characteristic depth, as for homogeneous cases. This is made clear in Fig. 9.22, which provides a graph of the normal force in relation to the maximum pull-off force in the classic JKR theory as a function of the normalized indentation depth. Equilibrium states corresponding to the dotted parts of the curves can only be realized for displacement-control. Additionally, Fig. 9.23 shows that the maximum pull-off force rises by increasing $k$, while the absolute value of the pull-off force decreases under displacement-controlled test conditions. 


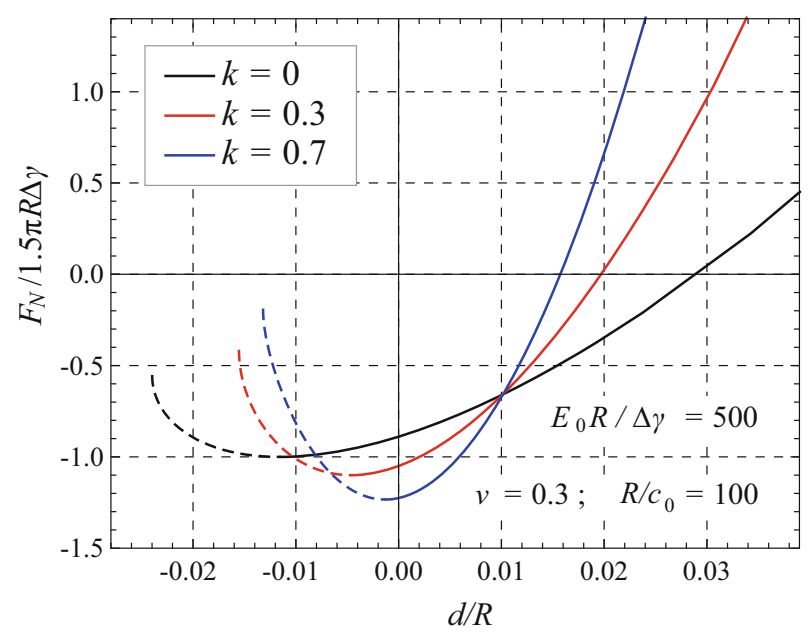

Fig. 9.23 Normal force as a function of the indentation depth in normalized representation for different values of $k$ and fixed characteristic depth $c_{0}$

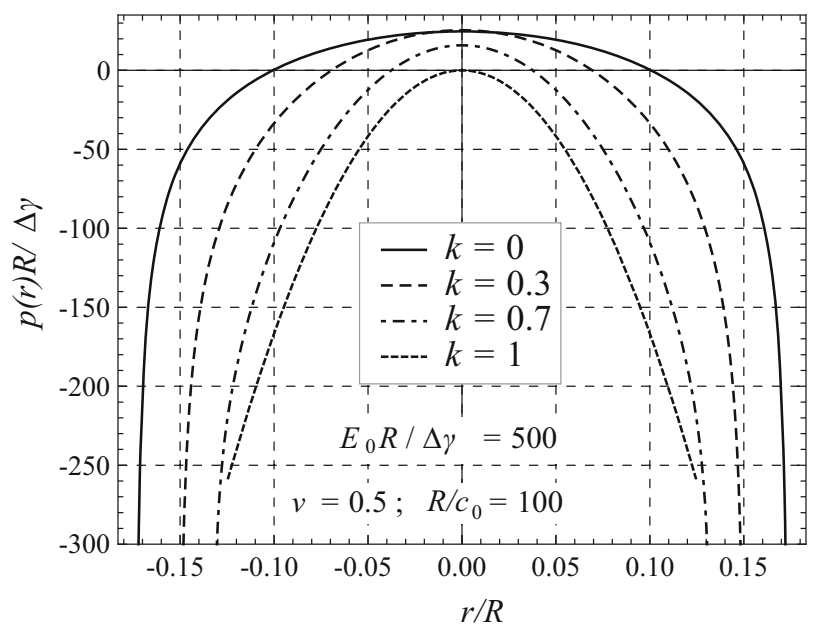

Fig. 9.24 Normalized pressure distribution in the contact area for a varying exponent $k$ and an incompressible material at a fixed characteristic depth $c_{0}$

The pressure distribution in the critical state, i.e., under the effect of the maximum pull-off force, is demonstrated for an incompressible material in Fig. 9.24. For the chosen parameters, the critical radius drops by increasing $k$. Figure 9.25 demonstrates that this property is not universally valid but instead dependent on the characteristic depth. Here we can also see that, for a fixed $k$, a decrease in the characteristic depth also leads to a decrease of the critical contact radius. Furthermore, 


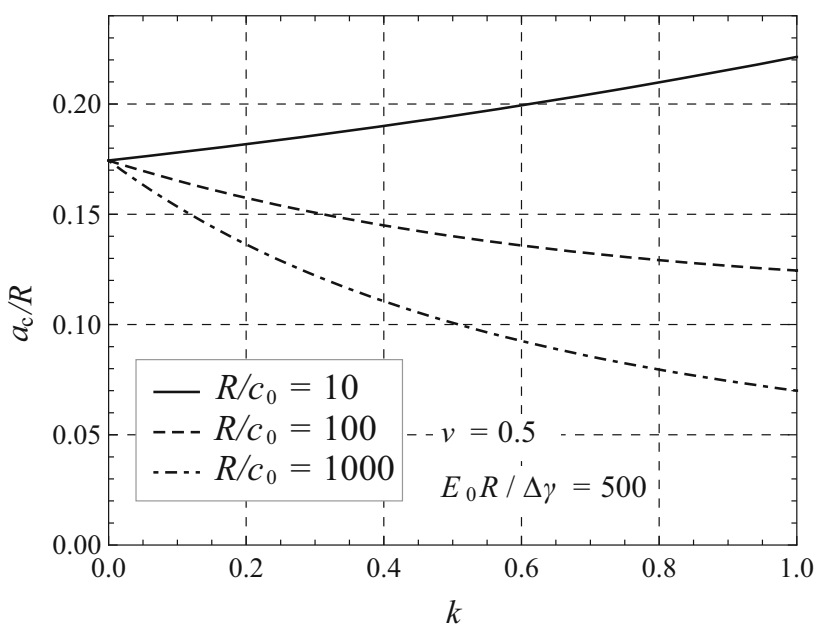

Fig. 9.25 Critical contact radius (fixed-load) as a function of $k$ for select characteristic depths $c_{0}$ and incompressible material

the maximum pressure is extremely sensitive to parameter variations and must be determined on a case-by-case basis. For the given parameters, the maximum pressure is reached at $k \approx 0.19$. In the Gibson medium $(k \rightarrow 1)$ the maximum pressure and, consequently, the (critical) indentation depth is zero.

As a last point, we have provided the following dimensionless equations:

$$
\begin{gathered}
\tilde{d}(\tilde{a})=\frac{1}{1-2 \tilde{C}(k)}\left(\tilde{a}^{2}-2 \tilde{C}(k) \tilde{a}^{\frac{1-k}{2}}\right) \quad \text { with } \tilde{d}:=\frac{d}{d_{c}} \text { and } \tilde{a}:=\frac{a}{a_{c}}, \\
\tilde{F}_{N}(\tilde{a})=\frac{1}{1-(3+k) \tilde{C}(k)}\left[\tilde{a}^{3+k}-(3+k) \tilde{C}(k) \tilde{a}^{\frac{3+k}{2}}\right] \quad \text { with } \tilde{F}_{N}:=\frac{F_{N}}{F_{c}},
\end{gathered}
$$

where the indentation depth, the normal force, and the contact radius are given in relation to their critical value. Jin et al. (2016) noted that the dimensionless forms of (9.57) depend solely on the exponent of the elastic inhomogeneity $k$.

\subsubsection{The Profile in the Form of a Power-Law}

Taking into consideration solutions (9.30) for the contact without adhesion, the universal superposition (9.49) provides the solution of the adhesive contact. For the adhesive normal contact of a rigid indenter with a profile in the shape of a power-law and an elastically inhomogeneous half-space, displayed schematically in Fig. 9.26, we obtain the following relationships between the indentation depth, contact radius, 
Fig. 9.26 Adhesive normal contact between a rigid indenter with a profile in the form of a power-law and an elastically inhomogeneous half-space

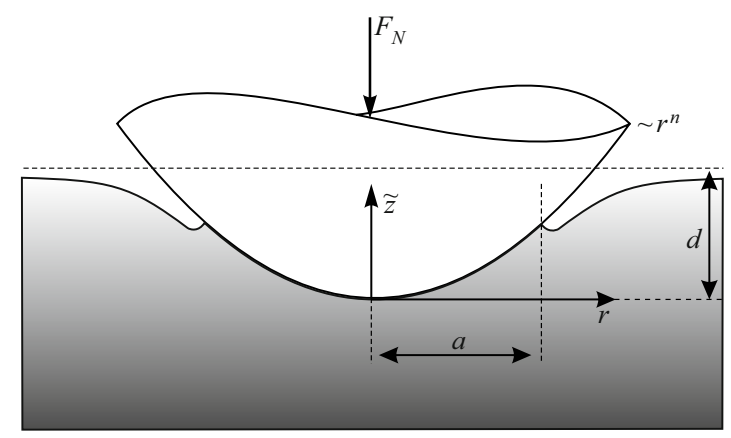

and normal force:

$$
\begin{aligned}
d(a) & =\kappa(n, k) A_{n} a^{n}-\sqrt{\frac{2 \pi \Delta \gamma c_{0}^{k} a^{1-k}}{E^{*} h_{N}(k, v)}}, \\
F_{N}(a) & =\frac{2 h_{N}(k, v) \kappa(n, k) n A_{n} E^{*} a^{n+k+1}}{(k+1)(n+k+1) c_{0}^{k}}-\sqrt{\frac{8 \pi h_{N}(k, v) \Delta \gamma E^{*} a^{3+k}}{(k+1)^{2} c_{0} k}} .
\end{aligned}
$$

The pressure distribution in the contact area and the normal surface displacement outside of the contact area are given by:

$$
\begin{aligned}
p(r ; a)= & \frac{h_{N}(k, v) \kappa(n, k) n A_{n} E^{*}}{2 \pi c_{0}^{k}} r^{n+k-1} \\
& \cdot\left[\mathrm{B}\left(\frac{1-k-n}{2}, \frac{1+k}{2}\right)-\mathrm{B}\left(\frac{r^{2}}{a^{2}} ; \frac{1-k-n}{2}, \frac{1+k}{2}\right)\right] \\
& -\sqrt{\frac{2 h_{N}(k, v) \Delta \gamma E^{*} a^{k-1}}{\pi c_{0} k}}\left[1-\left(\frac{r}{a}\right)^{2}\right]^{\frac{k-1}{2}}, \\
w(r ; a)= & \frac{\cos \left(\frac{k \pi}{2}\right) \kappa(n, k) A_{n} a^{n}}{\pi}\left(\mathrm{B}\left(\frac{a^{2}}{r^{2}} ; \frac{1+k}{2}, \frac{1-k}{2}\right)-\left(\frac{r}{a}\right)^{n} \mathrm{~B}\left(\frac{a^{2}}{r^{2}} ; \frac{1+k+n}{2}, \frac{1-k}{2}\right)\right] \\
& -\frac{\cos \left(\frac{k \pi}{2}\right)}{\pi} \sqrt{\frac{2 \pi \Delta \gamma c_{0}^{k} a^{1-k}}{E^{*} h_{N}(k, v)}} \mathrm{B}\left(\frac{a^{2}}{r^{2}} ; \frac{1+k}{2}, \frac{1-k}{2}\right) .
\end{aligned}
$$

The critical contact radii are determined by evaluating condition (9.48), which requires the slope of the equivalent profile at the contact edge as its input. Differentiation of (9.28) yields $g^{\prime}(a)=n \kappa(n, k) A_{n} a^{n-1}$, resulting in the following critical radii:

$$
a_{c}=\left(\frac{2 \pi \Delta \gamma c_{0}{ }^{k}}{h_{N}(k, v) E^{*} \tilde{C}(k)^{2} \kappa(n, k)^{2} n^{2} A_{n}{ }^{2}}\right)^{\frac{1}{2 n+k-1}} .
$$


Substituting (9.60) into (9.58) leads to critical indentation depths and forces,

$$
\begin{aligned}
d_{c}= & {\left[\left(\frac{2 \pi \Delta \gamma c_{0}{ }^{k}}{h_{N}(k, v) E^{*}}\right)^{n}\left(\frac{1}{\tilde{C}(k) n \kappa(n, k) A_{n}}\right)^{1-k}\right]^{\frac{1}{2 n+k-1}}\left(\frac{1-n \tilde{C}(k)}{n \tilde{C}(k)}\right), } \\
F_{c}= & 2\left(\frac{1-\tilde{C}(k)(n+k+1)}{\tilde{C}(k)(n+k+1)(k+1)}\right) \\
& \cdot\left[(2 \pi \Delta \gamma)^{n+k+1}\left(\frac{c_{0}{ }^{k}}{h_{N}(k, \nu) E^{*}}\right)^{2-n}\left(\frac{1}{\tilde{C}(k) n \kappa(n, k) A_{n}}\right)^{k+3}\right]^{\frac{1}{2 n+k-1}} .
\end{aligned}
$$

Jin et al. (2013) presented a detailed examination of the maximum pull-off force depending on the shape exponents $n$. For scaling reasons, the indenter shape was specified according to slightly modified function $f(r)=r^{n} /\left(n R^{n-1}\right)$ instead of (9.27). Normalizing their result to the maximum pull-off force of the classical JKR problem, they derived the following relationship:

$$
\begin{aligned}
\frac{F_{c}}{1.5 \pi \Delta \gamma R}= & \frac{2(1-k-2 n)}{3 \pi(n+k+1)(k+1)} \\
& \cdot\left[(2 \pi)^{n+k+1}\left(\frac{1-v^{2}}{h_{N}(k, v)}\right)^{2-n}\left(\frac{3+k}{2 \kappa(n, k)}\right)^{k+3}\right. \\
& \left.\cdot\left(\frac{R}{c_{0}}\right)^{k(n-2)}\left(\frac{E_{0} R}{\Delta \gamma}\right)^{n-2}\right]^{\frac{1}{2 n+k-1}},
\end{aligned}
$$

which is visualized for selected parameters and shape exponents in Fig. 9.27. It is clear to see that the maximum pull-off force changes linearly with $k$ only for the parabolic contact $(n=2)$ and otherwise exhibits a non-linear dependency.

Putting the indentation depth and normal force in relation to their critical values results in the following dimensionless representations, which depend solely on the exponents $k$ and $n$ (see Heß 2016a):

$$
\begin{aligned}
\tilde{d}(\tilde{a})= & \frac{1}{1-\tilde{C}(k) n}\left(\tilde{a}^{n}-n \tilde{C}(k) \tilde{a}^{\frac{1-k}{2}}\right) \quad \text { with } \tilde{d}:=\frac{d}{d_{c}} \text { and } \tilde{a}:=\frac{a}{a_{c}}, \\
\tilde{F}_{N}(\tilde{a})= & \frac{1}{1-\tilde{C}(k)(n+k+1)}\left[\tilde{a}^{n+k+1}-\tilde{C}(k)(n+k+1) \tilde{a}^{\frac{k+3}{2}}\right] \\
& \text { with } \tilde{F}_{N}:=\frac{F_{N}}{F_{c}} .
\end{aligned}
$$

\subsubsection{The Concave Paraboloid (Complete Contact)}

In the following, we will assume that a rigid, cylindrical, concave punch is initially pressed into the inhomogeneous half-space to the point of complete contact. During 


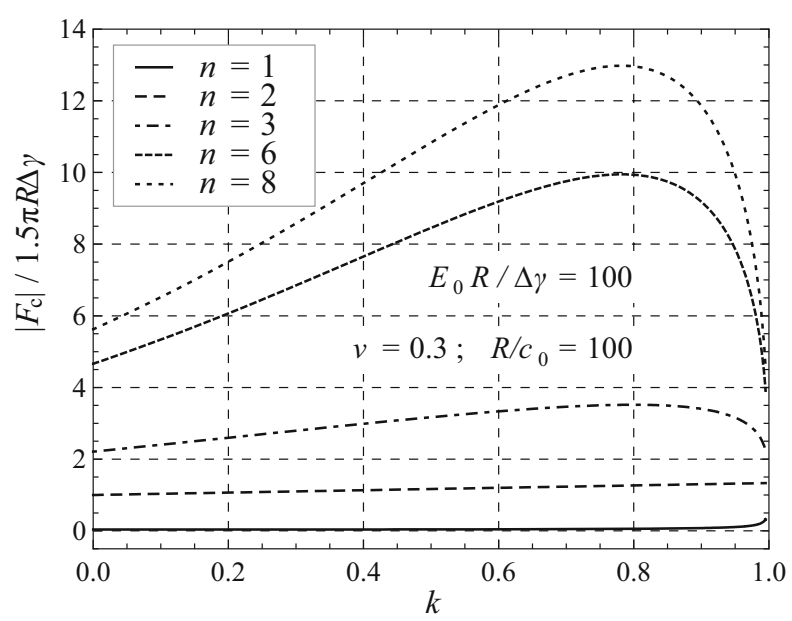

Fig. 9.27 Maximum pull-off force as a function of the exponent of the elastic inhomogeneity $k$ for different exponents of the power-law profile according to Jin et al. (2013)

the subsequent unloading, two competing mechanisms lead to the separation of the surfaces:

1. For slightly concave punch profiles, the separation of the punch is caused (as in convex profiles) by the stress singularity at the contact edge.

2. For highly concave punch profiles, the separation occurs in the center of the contact area because of too high tensile stresses, whose absolute values exceed the theoretical tensile strength (of the adhesion) $\sigma_{\text {th }}$.

The two states, whose accompanying quantities we denote by the indices "P" (Periphery) and "C" (Center), were examined in greater detail by Jin et al. (2013). The critical state, at which the separation at the edge occurs, satisfies the equilibrium condition (9.45). The displacement of the Winkler foundation is taken from the solution of the corresponding contact without adhesion, (9.36). By replacing $d$ by $d_{\mathrm{P}}$ we get:

$$
w_{1 D}(x)=d_{\mathrm{P}}-h_{0}+\frac{2 h_{0}}{k+1} \frac{x^{2}}{a^{2}} .
$$

The evaluation of condition (9.45) yields the critical indentation depth $d_{\mathrm{P}}$ at which the edge begins to separate:

$$
d_{\mathrm{P}}=-\sqrt{\frac{2 \pi \Delta \gamma c_{0}^{k} a^{1-k}}{h_{N}(k, v) E^{*}}}-h_{0} \frac{1-k}{1+k} .
$$

The remaining terms of the complete solution of the contact problem are obtained by simply inserting (9.65) into (9.34), taking into account that $d_{0}=d_{\mathrm{P}}-h_{0}$. Here, 
we restrict the presentation to the normal force and the pressure distribution:

$$
\begin{gathered}
F_{\mathrm{P}}(a)=-\sqrt{\frac{8 \pi h_{N}(k, v) \Delta \gamma E^{*} a^{3+k}}{(1+k)^{2} c_{0}^{k}}}-\frac{8 h_{N}(k, v) E^{*} h_{0} a^{1+k}}{(k+1)^{2}(k+3) c_{0}^{k}}, \\
p_{\mathrm{P}}(r ; a)=\frac{h_{N}(k, v) E^{*}}{\pi c_{0}^{k}\left(a^{2}-r^{2}\right)^{\frac{1-k}{2}}}\left[-\sqrt{\frac{2 \pi \Delta \gamma c_{0}^{k} a^{1-k}}{h_{N}(k, v) E^{*}}}-\frac{4 h_{0}}{(k+1)^{2}}\left(1-\frac{r^{2}}{a^{2}}\right)\right] .
\end{gathered}
$$

The first term of the critical force in (9.66) returns exactly the adhesive force of the flat punch according to (9.52). The second term results from the concave shape. Since both terms have the same sign, the maximum pull-off force of weakly concave profiles is always greater than the one of the flat punch contact.

For strongly concave profiles, the separation begins in the center of the contact area upon reaching the theoretical adhesive tensile strength. The critical indentation depth is gained from the condition $p(r=0)=-\sigma_{\text {th }}$, where once again, in the pressure distribution of the contact without adhesion $(9.34), d$ is replaced by $d_{\mathrm{C}}$. Therefore, we obtain:

$$
d_{\mathrm{C}}=-\frac{\pi c_{0}^{k} a^{1-k}}{h_{N}(k, v) E^{*}} \sigma_{\text {th }}+\frac{3+k^{2}}{(1+k)^{2}} h_{0},
$$

from which the normal force and the pressure distribution in the critical state follow as:

$$
\begin{aligned}
& F_{\mathrm{C}}(a)=-\frac{2 \pi a^{2}}{(1+k)} \sigma_{\mathrm{th}}+\frac{16 h_{N}(k, v) h_{0} E^{*} a^{k+1}}{(k+1)^{3}(k+3) c_{0}^{k}}, \\
& p_{\mathrm{C}}(a)=\frac{1}{\left(a^{2}-r^{2}\right)^{\frac{1-k}{2}}}\left[-a^{1-k} \sigma_{\mathrm{th}}+\frac{4 h_{N}(k, v) h_{0} E^{*}}{\pi(k+1)^{2} c_{0}^{k}} \frac{r^{2}}{a^{2}}\right] .
\end{aligned}
$$

For $k=0$, these solutions coincide with those of Waters et al. (2011).

\subsubsection{The Indenter Which Generates a Constant Adhesive Tensile Stress}

The optimal profile of a rigid, concave, cylindrical indenter is defined by the condition that, in the critical state, the constant tensile stresses in the contact area are (in absolute terms) equal to the theoretical adhesive tensile strength (see Gao and Yao 2004):

$$
p_{\text {opt }}(r)=-\sigma_{\text {th }} \quad \text { for } r \leq a,
$$

The maximum pull-off force is then

$$
F_{\mathrm{opt}}(a)=-\sigma_{\mathrm{th}} \pi a^{2}
$$

Determining the corresponding optimal shape is rendered particularly easy when accounting for the results from Sect. 9.1.7. Equations (9.39) and (9.40) determine 
the necessary surface displacement to generate a constant pressure in the contact area. Replacing $p_{0}$ with $-\sigma_{\text {th }}$ or, alternatively, $F_{N}$ by $F_{\text {opt }}$ according to (9.70) leads to the surface displacement of the considered problem:

$$
w_{\text {opt }}(r ; a)= \begin{cases}w_{c, \text { opt } 2} \mathrm{~F}_{1}\left(\frac{k-1}{2}, \frac{1+k}{2} ; 1 ; \frac{r^{2}}{a^{2}}\right) & \text { for } r \leq a, \\ w_{c, \text { opt }} \frac{1-k}{2}\left(\frac{a}{r}\right){ }^{1+k}{ }_{2} \mathrm{~F}_{1}\left(\frac{1+k}{2}, \frac{1+k}{2} ; 2 ; \frac{a^{2}}{r^{2}}\right) & \text { for } r>a,\end{cases}
$$

with

$$
w_{c, \mathrm{opt}}=-\frac{2 c_{0}^{k} \cos \left(\frac{k \pi}{2}\right) a^{1-k}}{h_{N}(k, v)(1-k) E^{*}} \sigma_{\mathrm{th}} .
$$

The optimal indenter shape $f_{\text {opt }}$ can basically be extracted from (9.71) and (9.72). Accounting for $f_{\mathrm{opt}}(r=0)=0$ returns:

$$
f_{\text {opt }}(r)=w_{c, \text { opt }}\left[1-{ }_{2} \mathrm{~F}_{1}\left(\frac{k-1}{2}, \frac{1+k}{2} ; 1 ; \frac{r^{2}}{a^{2}}\right)\right] .
$$

For the purposes of a graphical representation of the influence of the elastic inhomogeneity $k$ and the normalized characteristic depth $c_{0} / a$, it seems more appropriate to use the function shifted by the boundary value $f_{\mathrm{opt}}(a)$

$$
\tilde{f}_{\mathrm{opt}}(r):=f_{\mathrm{opt}}(r)-f_{\mathrm{opt}}(a)
$$

In Figs. 9.28 and 9.29 the optimal profile functions are plotted for different values of $k$ and $c_{0} / a$, according to the publication by Jin et al. (2013). For a fixed exponent

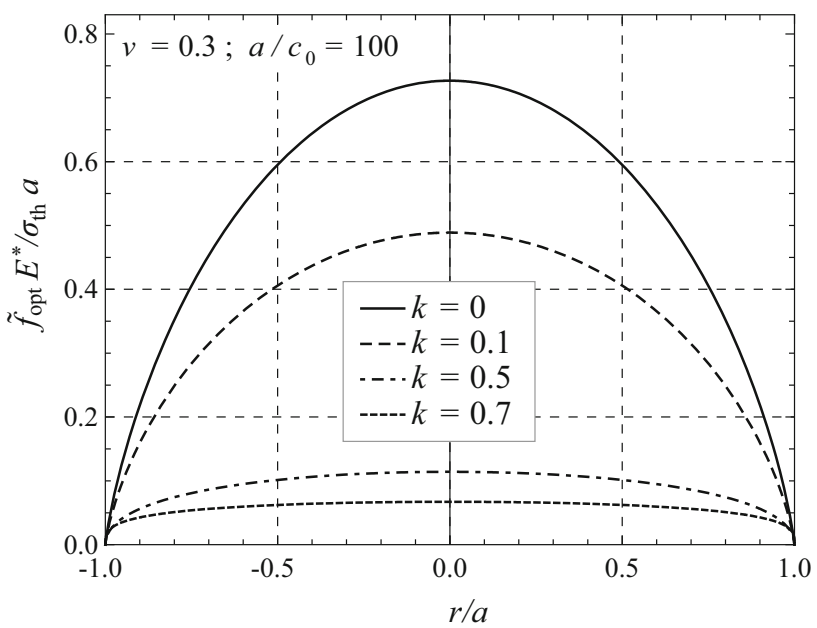

Fig.9.28 Optimal concave profile for a fixed characteristic depth $c_{0}$ and different set parameters $k$ 


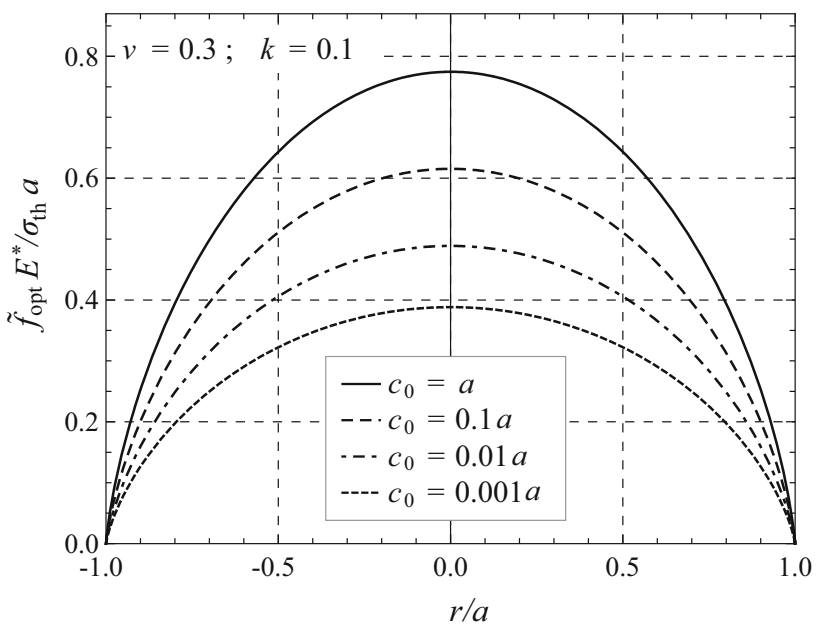

Fig. 9.29 Optimal concave profile for a fixed exponent of the elastic inhomogeneity $k=0.1$ and different characteristic depths $c_{0}$

$k$, a decrease of the characteristic depth $c_{0}$ causes a drop in the height of the concave section.

\subsection{Tangential Contact}

\subsubsection{Basis of Calculation and Restricting Assumptions}

In the following, we present selected solutions of tangential contact problems with partial slip between two bodies constructed of FGMs, according to (9.1). For both bodies we assume equal exponents $k$ and characteristic depths $c_{0}$. Furthermore, as in the classical theory of Cattaneo (1938) and Mindlin (1949) (see Chap. 4), we operate under the assumption of a decoupling of the normal and tangential contact, which is valid for the following material pairings:

1. Equal elastic materials: $v_{1}=v_{2}=: v$ and $E_{01}=E_{02}=: E_{0}$

2. One body is rigid and the other elastic with a Poisson's ratio equal to the Holl ratio: $E_{0 i} \rightarrow \infty$ and $v_{j}=1 /(2+k)$ with $i \neq j$

3. The Poisson's ratios of both materials are given by the Holl ratio: $v_{1}=v_{2}=$ $1 /(2+k)$

Note that, due to thermodynamic stability, the Holl ratio can only be fulfilled for positive $k$. If we assume that the bodies are initially pressed together by a normal force $F_{N}$ and subsequently (under a constant normal force) are subjected to a tangential force, then the contact area will be composed of an inner stick zone and an outer slip zone (see Fig. 9.30). 
Fig. 9.30 Tangential contact with partial slip between two elastically inhomogeneous bodies; the contact area is composed of an inner stick zone and an outer slip zone

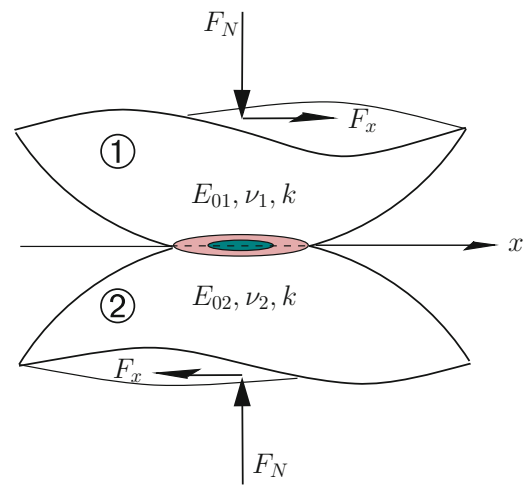

The boundary conditions are determined by the rigid-body translation of the points of the stick zone

$$
u(x, y)=\text { const. and } \quad v(x, y)=0 \text { for } 0<r<c
$$

and by the Coulomb law of friction

$$
\begin{aligned}
& |\vec{\tau}(r)|<\mu p(r) \quad \text { for } 0 \leq r<c, \\
& |\vec{\tau}(r)|=\mu p(r) \quad \text { for } c \leq r \leq a .
\end{aligned}
$$

In the slip zone, the tangential stresses must be additionally directed opposite to the relative tangential displacement of the surface points. With increasing tangential force, the stick zone shrinks until, finally, gross slip sets in. For the calculation of this problem using the generalized Ciavarella-Jäger theorem is recommended, according to which the tangential contact can be represented as the superposition of two normal contacts. For the tangential stress, the tangential force, and the relative tangential displacement $u^{(0)}$ between two remote material points-within the contacting bodies and far from the contact interface - the following is valid (see $\mathrm{He} ß$ 2016b):

$$
\begin{aligned}
\tau(r) & =\mu[p(r, a)-p(r, c)], \\
F_{x} & =\mu\left[F_{N}(a)-F_{N}(c)\right], \\
u^{(0)} & =\mu \alpha[d(a)-d(c)] .
\end{aligned}
$$

We have defined $\tau(r):=-\tau_{z x}(r)$ and assume that a tangential force in the $x$ direction only leads to tangential stresses in the same $x$-direction. $\alpha$ represents the ratio of normal stiffness to tangential stiffness:

$$
\alpha\left(k, v_{i}, E_{0 i}\right):=\frac{\frac{1}{h_{T}\left(k, v_{1}\right) E_{01}}+\frac{1}{h_{T}\left(k, v_{2}\right) E_{02}}}{\frac{1-v_{1}^{2}}{h_{N}\left(k, v_{1}\right) E_{01}}+\frac{1-v_{2}^{2}}{h_{N}\left(k, v_{2}\right) E_{02}}},
$$


where

$$
\begin{aligned}
& h_{T}(k, v)= \\
& \frac{2 \beta(k, v) \cos \left(\frac{k \pi}{2}\right) \Gamma\left(1+\frac{k}{2}\right)}{\left(1-v^{2}\right) \sqrt{\pi} C(k, v) \sin \left(\frac{\beta(k, v) \pi}{2}\right) \Gamma\left(\frac{3+k}{2}\right)+\beta(k, v)(1+v) \Gamma\left(1+\frac{k}{2}\right)},
\end{aligned}
$$

and the additional functional relationships are defined as an addendum to (9.5). With (9.77) we are able to solve the tangential contact between arbitrarily shaped bodies, provided the solutions of the corresponding normal contact problem are known. It is noted that tangential contacts can be solved in an equally simple fashion using the MDR (Heß and Popov 2016).

\subsubsection{Tangential Contact Between Spheres (Parabolic Approximation)}

Applying (9.77) to the (parabolic) contact of two equally elastically inhomogeneous spheres of radius $R$ leads to the following results:

$$
\begin{array}{ll}
\tau(r ; a, c)= & \text { 2h }(k, v) E_{0} \\
\mu \frac{h_{N}}{\pi\left(1-v^{2}\right) c_{0}^{k}(k+1)^{2} R} \begin{cases}\left(a^{2}-r^{2}\right)^{\frac{1+k}{2}}-\left(c^{2}-r^{2}\right)^{\frac{1+k}{2}} & \text { for } 0 \leq r \leq c, \\
\left(a^{2}-r^{2}\right)^{\frac{1+k}{2}} & \text { for } c<r \leq a,\end{cases} \\
F_{x}(c, a)=\mu F_{N}(a)\left[1-\left(\frac{c}{a}\right)^{k+3}\right], \\
u^{(0)}(c, a)=\mu \frac{h_{N}(k, v)}{\left(1-v^{2}\right) h_{T}(k, v)} d(a)\left[1-\left(\frac{c}{a}\right)^{2}\right],
\end{array}
$$

where $F_{N}(a)$ and $d(a)$ are taken from the solutions of the normal contact (9.26).

Figure 9.31 shows the values of the tangential stresses along the $x$-axis under the assumption that the normal force is kept constant for all selected exponents $k$ of the elastic inhomogeneity. The quantities are normalized to the maximum pressure $p_{0, H}$ and the contact radius $a_{H}$ of the Hertzian contact problem. It is clearly visible that, compared to the respective Hertzian contact radius (contact of homogeneous materials), the contact radii are smaller for negative exponents and greater for positive exponents. In contrast, the tangential stress is greater for negative exponents and vice-versa. However, this behavior of the stresses is only observed in the slip zone and not necessarily true for the entire contact area. The curves presented in Fig. 9.32 make it clear that gross slip sets in at a small tangential displacement $u^{(0)}$ for negative exponents, and at a substantially greater displacement for positive exponents. For ease of comparison, the quantities are normalized to the tangential displacement $u_{c \text {,hom }}^{(0)}$, which marks the onset of gross slip of elastically homogeneous materials. 


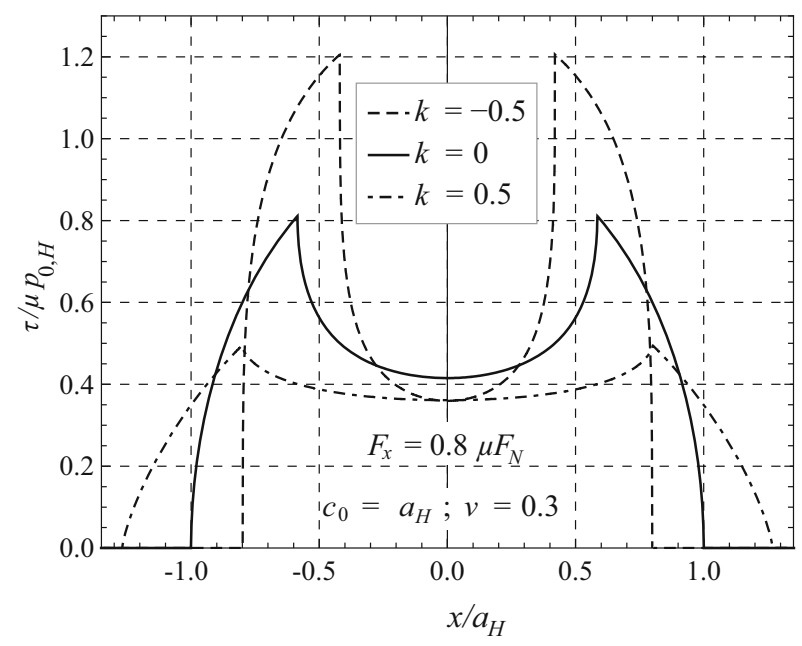

Fig. 9.31 Normalized tangential stresses along the $x$-axis for different exponents of the elastic inhomogeneity for set values of $F_{x}=0.8 \mu F_{N}, c_{0}=a_{H}$ and $v=0.3$

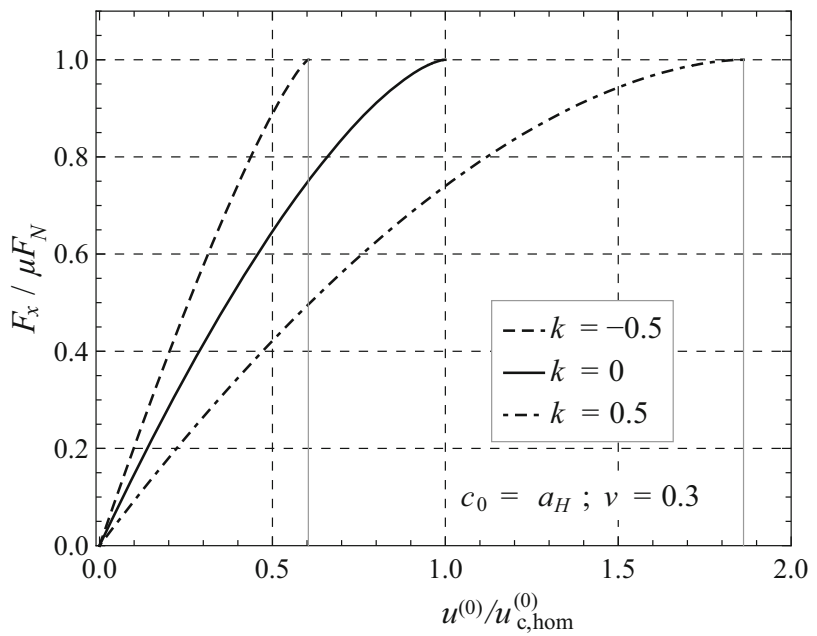

Fig. 9.32 Normalized tangential force as a function of the normalized tangential displacement for different exponents of the elastic inhomogeneity for set values of $c_{0}=a_{H}, v=0.3$ 


\subsubsection{Oscillating Tangential Contact of Spheres}

Two bodies with parabolically curved surfaces (curvature radii $R$ ) of the same elastically inhomogeneous material according to (9.1) are initially pressed together by a normal force, and subsequently (under a constant normal force) are subjected to the oscillating tangential force given in Fig. 9.33. Accordingly, the tangential force varies within the bounds $\pm 0.9 \mu F_{N}$ to avoid gross slip at every point in time.

The solutions of the contact problem for the initial rise to Point A on the load curve were covered in Sect. 9.3.2. Slip will occur, beginning at the edge of the contact, during a subsequent successive reduction of the tangential force. It is directed opposite to the initial slip and propagates inwards during the unloading process. In the new ring-shaped slip zone $b<r \leq a$, frictional stresses develop, which are again directed opposite to the initial frictional stresses. Therefore, a reduction in the tangential force leads to a change of the frictional stresses in the ring-shaped slip zone by $-2 \mu p(r)$. Yet since no slip occurs in the remaining contact area, all surface points in that domain are subjected to the same change in their tangential displacement. Therefore the change of the tangential force leads to changes of the tangential stresses and tangential displacements, which solve the contact problem formulated in Sect. 9.3.1. Thus, except for the factor " 2 " and a negative sign, the changes satisfy (9.77):

$$
\begin{aligned}
\Delta \tau(r) & =-2 \mu[p(r, a)-p(r, b)], \\
\Delta F_{x} & =-2 \mu\left[F_{N}(a)-F_{N}(b)\right], \\
\Delta u^{(0)} & =-2 \mu \alpha[d(a)-d(b)] .
\end{aligned}
$$

Fig. 9.33 Loading history of the tangential force: oscillation of the tangential force between the extreme values $\pm 0.9 \mu F_{N}$

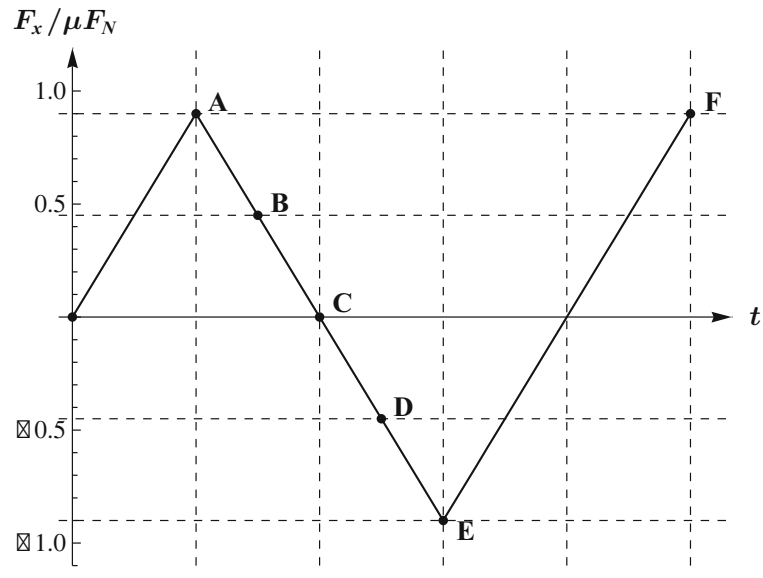


The absolute values result from the summation of the state variables in Point $\mathrm{A}$ and the changes (9.81). For the stresses, this leads to:

$$
\begin{aligned}
& \tau(r)= \\
& \mu \frac{2 h_{N}(k, v) E_{0}}{\pi\left(1-v^{2}\right) c_{0}^{k}(k+1)^{2} R} \\
& \cdot \begin{cases}-\left(a^{2}-r^{2}\right)^{\frac{1+k}{2}}+2\left(b^{2}-r^{2}\right)^{\frac{1+k}{2}}-\left(c^{2}-r^{2}\right)^{\frac{1+k}{2}} & \text { for } 0 \leq r \leq c, \\
-\left(a^{2}-r^{2}\right)^{\frac{1+k}{2}}+2\left(b^{2}-r^{2}\right)^{\frac{1+k}{2}} & \text { for } c<r \leq b, \\
-\left(a^{2}-r^{2}\right)^{\frac{1+k}{2}} & \text { for } b<r \leq a,\end{cases}
\end{aligned}
$$

and for the tangential force and the tangential displacements, this leads to:

$$
\begin{aligned}
& F_{x}=F_{A}-2 \mu F_{N}(a)\left[1-\left(\frac{b}{a}\right)^{k+3}\right] \text { with } F_{A}=\mu F_{N}(a)\left[1-\left(\frac{c}{a}\right)^{k+3}\right], \\
& u^{(0)}=u_{A}^{(0)}-2 \mu \alpha d(a)\left[1-\left(\frac{b}{a}\right)^{2}\right] \quad \text { with } \quad u_{A}^{(0)}=\mu \alpha d(a)\left[1-\left(\frac{c}{a}\right)^{2}\right] .
\end{aligned}
$$

$F_{A}$ and $u_{A}^{(0)}$ represent the load and displacement quantities in Point $\mathrm{A}$, and $c$ is the radius of the corresponding stick zone. For the selected exponents of the elastic inhomogeneity $k=0.5$ and $k=-0.5$, Figs. 9.34 and 9.35 display the tangential stress distribution along the $x$-axis for the characteristic points A through $\mathrm{E}$ of the unloading curve (see Fig. 9.33). In both cases, the same normal force was applied and the representation was normalized to the radius $a_{H}$ of the Hertzian contact corresponding to this normal force. For positive $k$, the increase of the contact

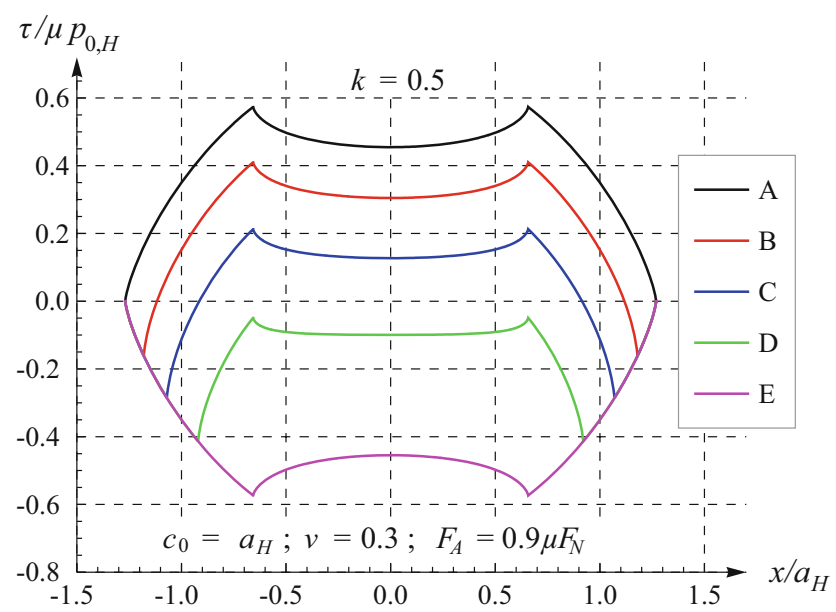

Fig. 9.34 Curve of the normalized tangential stresses along the $x$-axis for different points of the load curve from Fig. 9.33 and positive exponents of the elastic inhomogeneity $k=0.5$ 


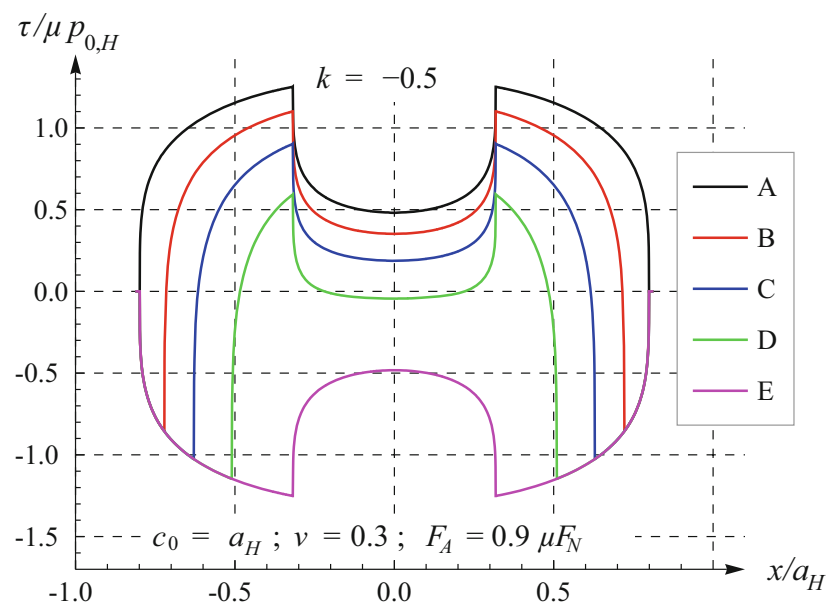

Fig. 9.35 Curve of the normalized tangential stresses along the $x$-axis for different points of the load curve from Fig. 9.33 and negative exponents of the elastic inhomogeneity $k=-0.5$

area is coupled with a decrease in the tangential stresses. On the other hand, negative exponents reduce the contact area, resulting in an increase of the tangential stresses compared to the homogeneous case $k=0$. However, this effect is extremely sensitive to changes in the characteristic depth and can even manifest in the inverse behavior. In Figs. 9.34 and 9.35 it is assumed that $c_{0}=a_{H}$. The characteristic difference in the shape of the curves for positive and negative exponents remain unaffected. The dependency of the stress distribution on the loading history is demonstrated best at the load point $\mathrm{C}$. Here, non-zero tangential stresses arise, even though no external tangential force is applied.

The solutions for the load curve from $\mathrm{E}$ to $\mathrm{F}$ (see Fig. 9.33) follow in complete analogy to the solution from A to E.

Using (9.83), the tangential force can be expressed as a function of the tangential displacement for the unloading path from $\mathrm{A}$ to $\mathrm{E}$ and in the same manner for the subsequent loading path from $\mathrm{E}$ to $\mathrm{F}$. These are:

$$
\begin{aligned}
& \mathrm{A} \rightarrow \mathrm{E}: F_{x}=F_{A}-2 \mu F_{N}(a)\left[1-\left(1+\frac{u^{(0)}-u_{A}^{(0)}}{2 \alpha \mu d(a)}\right)^{\frac{k+3}{2}}\right], \\
& \mathrm{E} \rightarrow F: F_{x}=-F_{A}+2 \mu F_{N}(a)\left[1-\left(1-\frac{u^{(0)}+u_{A}^{(0)}}{2 \alpha \mu d(a)}\right)^{\frac{k+3}{2}}\right] .
\end{aligned}
$$




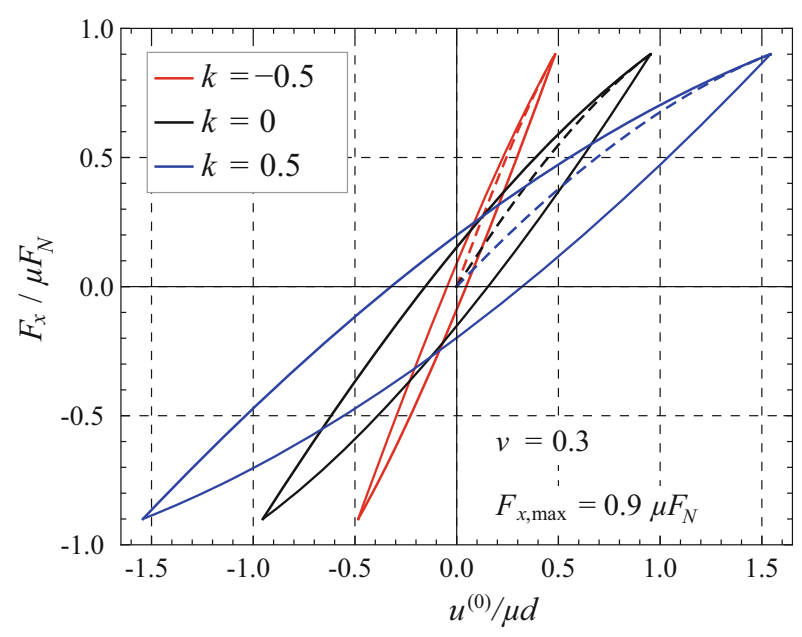

Fig. 9.36 Hysteresis loops as a result of an oscillating tangential contact for different exponents of the elastic inhomogeneity

where the tangential displacement in Point A depends on the corresponding tangential force according to:

$$
u_{A}^{(0)}=\mu \alpha d(a)\left[1-\left(1-\frac{F_{A}}{\mu F_{N}}\right)^{\frac{2}{k+3}}\right] .
$$

Equations (9.84) characterize a hysteresis curve, a graphical representation of which is given in Fig. 9.36 for different values of the exponent $k$. In the chosen normalization, it is quite visible that, compared to the homogeneous case, the gradient of the hysteresis is greater for negative exponents and smaller for positive exponents. Additionally, an increasing $k$ is connected with an increase of the area enclosed by the hysteresis loop and, therefore, the dissipated energy per loading cycle. An explicit calculation of the energy dissipated per cycle yields:

$$
\begin{aligned}
\Delta W=\frac{4(k+3)^{2}(k+1) \mu^{2} F_{N}^{2} c_{0}^{k}}{(k+5) h_{T}(k, v) E_{0} a^{k+1}} \\
\quad \cdot 1-\left(1-\frac{F_{A}}{\mu F_{N}}\right)^{\frac{k+5}{k+3}} \\
\left.\quad-\frac{k+5}{2(k+3)} \frac{F_{A}}{\mu F_{N}}\left(1+\left(1-\frac{F_{A}}{\mu F_{N}}\right)^{\frac{2}{k+3}}\right)\right] .
\end{aligned}
$$


The special case of elastically homogeneous bodies $(k=0)$ leads to the result derived by Mindlin and Deresiewicz (1953):

$$
\Delta W=\frac{18 \mu^{2} F_{N}^{2}}{5 G^{*} a}\left[1-\left(1-\frac{F_{A}}{\mu F_{N}}\right)^{\frac{5}{3}}-\frac{5}{6} \frac{F_{A}}{\mu F_{N}}\left(1+\left(1-\frac{F_{A}}{\mu F_{N}}\right)^{\frac{2}{3}}\right)\right]
$$

\section{References}

Aleynikov, S.M.: Spatial contact problems in Geotechnics: boundary-element method. Springer, Heidelberg, pp 55-83 (2011). ISBN 978-3-540-25138-5

Argatov, I., Heß, M., Popov, V.L.: The extension of the method of dimensionality reduction to layered elastic media. Z. Angew. Math. Mech. 98(4), 622-634 (2018)

Awojobi, A.O., Gibson, R.E.: Plane strain and axially-symmetric problems of a linearly nonhomogeneous elastic half-space. Q. J. Mech. Appl. Math. 26(3), 285-302 (1973)

Boesel, L.F., Greiner, C., Arzt, E., Del Campo, A.: Gecko-inspired surfaces: a path to strong and reversible dry adhesives. Adv. Mater. 22(19), 2125-2137 (2010)

Booker, J.R., Balaam, N.P., Davis, E.H.: The behavior of an elastic non-homogeneous half-space. Part II-circular and strip footings. Int. J. Numer. Anal. Methods. Geomech 9(4), 369-381 (1985)

Brown, P.T., Gibson, R.E.: Surface settlement of a deep elastic stratum whose modulus increases linearly with depth. Can. Geotech. J. 9(4), 467-476 (1972)

Cattaneo, C.: Sul contatto di due corpi elastici: distribuzione locale degli sforzi. Rendiconti Dell'accademia Nazionale Dei Lincei 27, 342-348, 434-436, 474-478 (1938)

Chen, S., Yan, C., Zhang, P., Gao, H.: Mechanics of adhesive contact on a power-law graded elastic half-space. J. Mech. Phys. Solids 57(9), 1437-1448 (2009)

Fabrikant, V.I., Sankar, T.S.: On contact problems in an inhomogeneous half-space. Int. J. Solids Struct. 20(2), 159-166 (1984)

Fröhlich, O.K.: XI Das elastische Verhalten der Böden. In: Druckverteilung im Baugrunde, pp. 86-108. Springer, Vienna (1934)

Gao, H., Yao, H.: Shape insensitive optimal adhesion of nanoscale fibrillar structures. Proc. Natl. Acad. Sci. U.S.A. 101(21), 7851-7856 (2004)

Giannakopoulos, A.E., Pallot, P.: Two-dimensional contact analysis of elastic graded materials. J. Mech. Phys. Solids 48(8), 1597-1631 (2000)

Giannakopoulos, A.E., Suresh, S.: Indentation of solids with gradients in elastic properties: Part II. Axi-symmetric indentors. Int. J. Solids Struct. 34(19), 2393-2428 (1997)

Gibson, R.E.: Some results concerning displacements and stresses in a non-homogeneous elastic half-space. Geotechnique 17(1), 58-67 (1967)

Gorb, S., Varenberg, M., Peressadko, A., Tuma, J.: Biomimetic mushroom-shaped fibrillar adhesive micro-structure. J. R. Soc. Interface 4(13), 271-275 (2007)

Heß, M.: A simple method for solving adhesive and non-adhesive axi-symmetric contact problems of elastically graded materials. Int. J. Eng. Sci. 104, 20-33 (2016a)

Heß, M.: Normal, tangential and adhesive contacts between power-law graded materials. Presentation at the Workshop on Tribology and Contact Mechanics in Biological and Medical Applications, TU-Berlin, 14.-17. Nov. 2016. (2016b)

Heß, M., Popov, V.L.: Method of dimensionality reduction in contact mechanics and friction: a user's handbook. II. Power-law graded materials. Facta Univ. Ser. Mech. Eng. 14(3), 251-268 (2016)

Holl, D.L.: Stress transmission in earths. Highway Res. Board Proc. 20, 709-721 (1940)

Jin, F., Guo, X., Zhang, W.: A unified treatment of axi-symmetric adhesive contact on a power-law graded elastic half-space. J. Appl. Mech. 80(6), 61024 (2013) 
Jin, F., Zhang, W., Wan, Q., Guo, X.: Adhesive contact of a power-law graded elastic half-space with a randomly rough rigid surface. Int. J. Solids Struct. 81, 244-249 (2016)

Jitcharoen, J., Padture, P.N., Giannakopoulos, A.E., Suresh, S.: Hertzian-crack suppression in ceramics with elastic-modulus-graded surfaces. J. Am. Ceram. Soc. 81(9), 2301-2308 (1998)

Lee, D., Barber, J.R., Thouless, M.D.: Indentation of an elastic half-space with material properties varying with depth. Int. J. Eng. Sci. 47(11), 1274-1283 (2009)

Liu, Z., Meyers, M.A., Zhang, Z., Ritchie, R.O.: Functional gradients and heterogeneities in biological materials: design principles, functions, and bioinspired applications. Prog. Mater. Sci. 88, 467-498 (2017)

Mehrali, M., Shirazi, F.S., Mehrali, M., Metselaar, H.S.C., Kadri, N.A.B., Osman, N.A.A.: Dental implants from functionally graded materials. J. Biomed. Mater. Res. A 101(10), 3046-3057 (2013)

Mindlin, R.D.: Compliance of elastic bodies in contact. J. Appl. Mech. 16(3), 259-268 (1949)

Mindlin, R.D., Deresiewicz, H.: Elastic spheres in contact under varying oblique forces. J. Appl. Mech. 20, 327-344 (1953)

Miyamoto, Y., Kaysser, W.A., Rabin, B.H., Kawasaki, A., Ford, R.G.: Functionally graded materials: design, processing and applications. Kluwer Academic Publishers, Boston, Dordrecht, London (1999)

Peisker, H., Michels, J., Gorb, S.N.: Evidence for a material gradient in the adhesive tarsal setae of the ladybird beetle Coccinella septempunctata. Nat. Commun. 4, 1661 (2013)

Rostovtsev, N.A.: An integral equation encountered in the problem of a rigid foundation bearing on non-homogeneous soil. J. Appl. Math. Mech. 25(1), 238-246 (1961)

Rostovtsev, N.A.: On certain solutions of an integral equation of the theory of a linearly deformable foundation. J. Appl. Math. Mech. 28(1), 127-145 (1964)

Selvadurai, A.P.S.: The analytical method in geomechanics. Appl. Mech. Rev. 60(3), 87-106 (2007)

Sola, A., Bellucci, D., Cannillo, V.: Functionally graded materials for orthopedic applications-an update on design and manufacturing. Biotechnol. Adv. 34(5), 504-531 (2016)

Suresh, S.: Graded materials for resistance to contact deformation and damage. Sci. Compass Rev. 292(5526), 2447-2451 (2001)

Suresh, S., Olsson, M., Giannakopoulos, A.E., Padture, N.P., Jitcharoen, J.: Engineering the resistance to sliding-contact damage through controlled gradients in elastic properties at contact surfaces. Acta. Mater. 47(14), 3915-3926 (1999)

Waters, J.F., Gao, H.J., Guduru, P.R.: On adhesion enhancement due to concave surface geometries. J. Adhes. 87(3), 194-213 (2011)

Willert, E.: Dugdale-Maugis adhesive normal contact of axi-symmetric power-law graded elastic bodies. Facta Univ. Ser. Mech. Eng. 16(1), 9-18 (2018)

Willert, E., Popov, V.L.: Adhesive tangential impact without slip of a rigid sphere and a power-law graded elastic half-space. Z. Angew. Math. Mech. 97(7), 872-878 (2017a)

Willert, E., Popov, V.L.: The oblique impact of a rigid sphere on a power-law graded elastic halfspace. Mech. Mater. 109, 82-87 (2017b) 
Open Access This chapter is licensed under the terms of the Creative Commons Attribution 4.0 International License (http://creativecommons.org/licenses/by/4.0/), which permits use, sharing, adaptation, distribution and reproduction in any medium or format, as long as you give appropriate credit to the original author(s) and the source, provide a link to the Creative Commons license and indicate if changes were made.

The images or other third party material in this chapter are included in the chapter's Creative Commons license, unless indicated otherwise in a credit line to the material. If material is not included in the chapter's Creative Commons license and your intended use is not permitted by statutory regulation or exceeds the permitted use, you will need to obtain permission directly from the copyright holder.

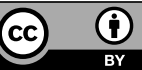

